\title{
Real-time dynamics of spin-dependent transport through a double-quantum-dot Aharonov-Bohm interferometer with spin-orbit interaction
}

\author{
Matisse Wei-Yuan Tu, ${ }^{1}$ Amnon Aharony, ${ }^{2}, 3$, 田 Wei-Min Zhang, ${ }^{1}$, \\ ${ }^{1}$ Department of Physics, National Cheng Kung University, Tainan 70101, Taiwan \\ ${ }^{2}$ Physics Department, Ben Gurion University, Beer Sheva 84105, Israel \\ ${ }^{3}$ Raymond and Beverly Sackler School of Physics and Astronomy, Tel Aviv University, Tel Aviv 69978, Israel
}

\begin{abstract}
The spin-resolved non-equilibrium real-time electron transport through a double-quantum-dot (DQD) Aharonov-Bohm (AB) interferometer with spin-orbit interaction (SOI) is explored. The SOI and $\mathrm{AB}$ interference in the real-time dynamics of spin transport is expressed by effective magnetic fluxes. Analytical formulae for the time-dependent currents, for initially unpolarized spins, are presented. In many cases, there appear spin currents in the electrodes, for which the spins in each electrode are polarized along characteristic directions, pre-determined by the SOI parameters and by the geometry of the system. Special choices of the system parameters yield steady-state currents in which the spins are fully polarized along these characteristic directions. The time required to reach this steady state depends on the couplings of the DQD to the leads. The magnitudes of the currents depend strongly on the SOI-induced effective fluxes. Without the magnetic flux, the spin-polarized current cannot be sustained to the steady states, due to the phase rigidity for this system. For a non-degenerate DQD, transient spin transport can be produced by the sole effects of SOI. We also show that one can extract the spin-resolved currents from measurements of the total charge current.
\end{abstract}

PACS numbers: 72.25.Dc,75.70.Tj,72.25.Rb,85.35.-p

\section{INTRODUCTION}

Electron interference in nanoscale quantum transport systems has long been a focus of intensive research. Of particular interest are the Aharonov-Bohm $(\mathrm{AB})^{\underline{1}}$ and the Aharonov-Cahser (AC) 2 effects, associated with the two fundamental degrees of freedom of an electron, namely, the charge and the spin. By tuning externally applied fields, one is able to modulate these interference effects and thus affect the quantum transport properties. Interesting results of coherence modulation have been found from the studies of stationary properties of mesoscopic interferometer systems. Dynamical responses of interference devices to periodically applied driving fields have also caught attention, due to their potential in applications. In addition, there is a rising interest in the real time dynamics of the charge and spin transport in such devices. This is relevant to temporal operations of quantum devices and also to the understanding of various physical processes. More and more attention is thus paid to the transient evolution of coherent electron transport. Naturally, the effects of interference on the transient dynamics of non-equilibrium transport is an important issue. In this paper, we study the dynamical evolution of electron transport through a double-quantum-dot (DQD) Aharonov-Bohm interferometer with spin-orbit interaction (SOI).

Coherence of the electron's orbital motion underlies the conductance oscillation in the applied magnetic flux, enclosed by low-dimensional electronic systems ${ }^{\underline{3}-\underline{6}}$ Studies of $\mathrm{AB}$ oscillations in $\mathrm{AB}$ interferometers with quantum dots have been realized in experiments ${ }^{7-10}$ Analogous to this $\mathrm{AB}$ oscillation, systems where the SOI is present exhibit conductance oscillations in the SOI strength, known as AC oscillations $\stackrel{11}{\underline{1}}$ Signatures of the
AC effects have also been observed in experiments $\underline{12-15}$ Besides interference effects in ring-shaped structures, SOI in nanoelectronic systems in general is known to have an important role in spintronics $\frac{16}{16}$ An important task in spintronics is to generate spin-polarized currents.

An early initiative in spintronics is the proposal of the spin-field-effect transistor by Datta and Das, that combined the SOI with ferromagnets $\frac{17}{17}$ Optical spin injection into ferromagnets for generating spin-polarized currents was experimentally implemented $\stackrel{18}{ }$ Electrical spin injection from ferromagnets to semiconductors was also realized. $\stackrel{19}{ }$ Spin-polarized currents can also be generated using magnetic tunnel junctions $\stackrel{20-23}{\underline{2}}$ Impedance mismatch between ferromagnets and semiconductors hinders efficient operation of spin injection, $\stackrel{24}{\rightleftharpoons}$ whose solution requires special techniques ${ }^{25}$ Generating spinpolarized currents without the use of ferromagnets, but with tunable SOI, is an alternative option. There are two kinds of SOI in mesoscopic electronic structures receiving special attentions, namely, the Dresselhaus SOI 26 and the Rashba SOI $\stackrel{27}{2}$ The former is a property of crystal structures that lack inversion symmetry in their unit cells. The latter, induced by the asymmetry in externally applied confinement potential, can be controlled by tuning this external electric field. The tunability of the Rashba SOI strength has been demonstrated experimentally, $\stackrel{12,13,28,29}{2}$ making the utilisation of SOI for generating spin-polarized current viable.

The simplest system that exhibits both the $\mathrm{AB}$ and the AC interference phenomena is a single loop. The loop is threaded by a magnetic flux and an electron can flip its spins as it tunnels along the loop. By attaching current leads to the loop, transport properties can be investigated. Spin interference effects on the electron transport through this kind of structures have been 
widely investigated. Many papers consider generating spin-polarized currents in such systems. These cover the modulation of conductance in one-dimensional and also two-dimensional circular rings,$\underline{30-33}$ and the effects of the coupling between the DQD and the leads on spindependent transport 34.35 Alternative system geometries, like polygons, have also been studied ${ }^{36-38}$ Instead of using just two leads, results from attaching three leads to the ring, mimicking a Stern-Gerlach experiment, have

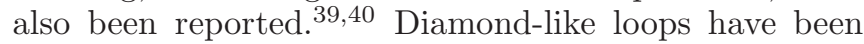
found to exhibit fully polarized spin currents $\stackrel{41}{\underline{43}}$ SOI in parallel DQD with inter-dot tunnel couplings have been considered $\underline{44}-\underline{47}$ Though interference is mostly effective at low temperatures, results from a high temperature single-channel ring are also analyzed $\underline{\underline{48}}$ Furthermore, electron-electron interactions have been studied in rings with $\mathrm{SOI} \stackrel{49,50}{50}$ Bides focusing on the timeindependent aspect, time-periodical varying SOI has attracted attention, $, 1,52$ as spin pumping devices.

Apart from this, much effort has been poured into the research of time-dependent electron transport through nanojunctions. Experimentally, time-resolved transport measurements have been implemented $\underline{\underline{53}-55}$ Theoretically, a multitude of approaches, focusing on many different aspects, has been devoted to understand the realtime electron dynamics in quantum transport $\underline{\underline{56}} \underline{\underline{68}}$ Realtime dynamics concerning spin-resolved currents have also been reported. By solving the time-dependent Schrödinger equation, the spin-resolved time evolution of the electron wave function in a ring with an oscillating

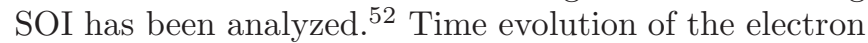
wave functions with different spins has also been considered in quantum well structures $\underline{69}$ Applying a method for Green functions propagating in time, $\underline{\underline{60}}$ transient spindependent currents through a single-level dot, without a loop structure, has been studied. $\stackrel{70}{\underline{70}}$

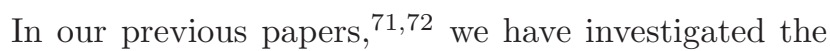
transient electron dynamics in a spinless $\mathrm{DQD} A \mathrm{AB}$ interferometer, based on a master equation formalism. $\underline{66.67}$ An earlier work had studied the steady states of a similar system with spins and SOI $\underline{43}$ Here we study the transient dynamics of spin-dependent transport in such a system. There are clear motivations to pursue such a study. First, spin polarization functions are closely related to functioning of flying spin qubits $\underline{\underline{43}}$ The dynamics of polarization processes is thus quintessential to the processing of quantum spin information in real time. Moreover, to coordinate the clocking of integrated spintronic circuits, the timing of the generation of spin-polarized currents as part of the circuit is indispensable. Closely examining the transient spin-resolved currents is an initiative toward these matters. Second, the ability of the targeted system to attain full spin filtering has been proved in the steady state. $\stackrel{43}{ }$ This makes it obviously worthy to explore its spin transport dynamics. Third, the interferometer possesses tunable coherent properties. It is therefore embedded with rich interference phenomena involving both charge and spin degrees of freedom.
In this paper, we address the following essential questions that are common to many devices designated for the generation of spin-polarized currents, a primary task in spintronics. These questions are: (i) What are the factors that determine the spin polarization directions? how do they change in time? (ii) What are the factors that determine the magnitude of currents of specific spins? (iii) How fast are the fully polarized currents reached? How do we control this temporal pace? Besides all these of operating the device, there is still an important question in terms of basic scientific research, namely, (iv) What are the physical mechanisms that lie behind the answers of the above questions?

The target system in this paper is illustrated in Fig. 1. We apply the nonequilibrium Green function technique (NEGF) for the calculation of spin-resolved real-time currents. We adopt the prescription for spin transformation along electron tunneling paths given in Ref. 73], and used in 43. Fully spin-polarized currents have been obtained in the steady-state limit using the spin filter conditions given in Ref. [43] (see sections II] and III). The spin-independent real-time total charge current is found to exhibit the universal behavior pointed out in Ref. 74 for rings with SOI (see discussions in Sec. III). In addition to discussing how different parameters of the system affect transient spin transport processes, we also provide instructions to extract the spin-polarized currents from the experimentally more accessible spin-independent total charge currents.

These investigations provide brief answers for our target system to the questions proposed above. The polarization directions of the currents in each of the two electrodes do not change in time and are pre-determined solely by the SOI parameters of the system. These parameters include the bonding geometry, adjustable in device fabrication, and the Rashba SOI strength, controllable by the external electric field. The applied magnetic flux, though found to be necessary in sustaining the spin polarization of the currents in the steady-state limit, plays no role in the determination of the polarization directions. However, the effective fluxes, composed of the applied magnetic flux and the SOI-induced phase, efficiently modulate the magnitudes of the polarized currents throughout the time. The couplings between the DQD and the electrodes then largely determine the times to reach the final stable polarizations. These consequences can be comprehended from the simple picture of two-path spinless interference of the two-terminal setup, based on the connection between the present spinful system and its spinless counterpart.

The paper is organized as follows. In Sec. [I] we analytically analyze the real-time transport through a DQD $\mathrm{AB}$ interferometer with SOI. In Sec. [IA we first introduce our model with a description of its SOI features. In Sec. IIB, we utilize the characteristic spinors of the SOIinduced unitary spin rotations, to show that the Hamiltonian of the target system can be decomposed into two commuting components, one for spin-up states and the 
other for spin-down states. Based on this decomposition, in Sec. IIC, we deduce the main results about spin polarization properties directly on the level of the Hamiltonian. Obtaining fully spin-polarized currents using the conditions given in Ref. [43] is also shown. In Sec. IID. the nonequilibrium formalism based on the master equation of the density matrix of the quantum-dot system is applied to the target system of a DQD AB interferometer with the SOI introduced in Sec. IA For the purpose of tackling the dynamics purely induced by SOI, the central area is initially prepared with no excess electrons. In this case, the connection of the present formalism with the standard Keldysh Green function technique is explicitly provided. To demonstrate the functioning of different physical factors behind spin-polarized transport with concrete examples, we take the commonly assumed wide-band limit for specific calculations. In Sec. III we first take the steady-state limit to reassure the reproduction of fully spin-polarized currents. We also analyze the situation when the setup of the system deviates from these conditions. This is followed by instructions for extracting the spin-polarized transmission from the spinindependent total transmission (which is much more accessible experimentally). Section IV is divided into three parts. In Sec. IVA we focus on the dynamics of getting fully spin-polarized currents. In Sec. IVB general parameters are explored to understand the transport of spins under the influence of charge and spin interferences. In addition, utilizing the results from Sec. III, we also devise similar ideas for extracting the spin-polarized currents from the spin-independent total charge currents in Sec. IVC. Conclusions and a summary are given in Sec. V.

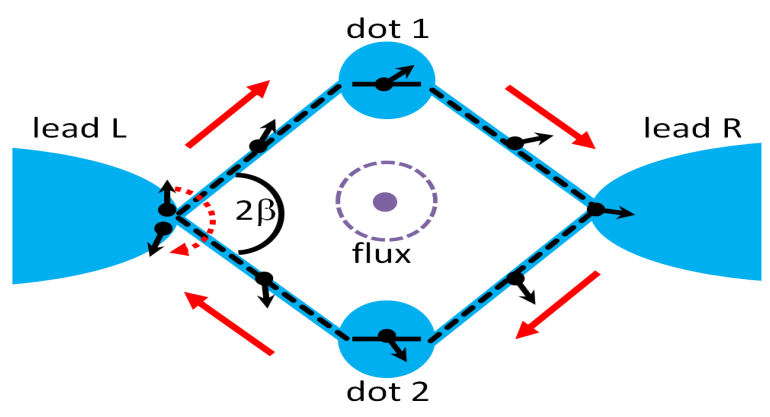

FIG. 1: (color online) A sketch of a DQD AB interferometer with SOI. Each of the two dots contains one charge state with a spin. The dots are placed in parallel between two leads $L$ and $R$. A magnetic flux threads through the loop formed by the two dots and the two leads. The electron spin rotates as it tunnels along the loop, due to the SOI. The angle between the two paths is $2 \beta$.

\section{REAL-TIME TRANSPORT THROUGH A DQD AB INTERFEROMETER WITH SOI}

\section{A. The model}

The DQD interferometer which we consider here is schematically presented in Fig. 1. It is composed of three parts, the DQD, the two electron reservoirs on the left and on the right and the tunneling between the DQD and the electrodes. The electron reservoirs are free from SOI. The total Hamiltonian is then generally given by

$$
\mathcal{H}=\mathcal{H}_{\mathrm{S}}+\mathcal{H}_{\mathrm{E}}+\mathcal{H}_{\mathrm{T}}
$$

Here we focus only on SOI and ignore Zeeman splitting. Explicitly, the central system Hamiltonian is specified to,

$$
\mathcal{H}_{\mathrm{S}}=\sum_{\sigma} \sum_{i=1}^{2} E_{i} a_{i \sigma}^{\dagger} a_{i \sigma} .
$$

The DQD system is spin-degenerate with $E_{i}$ 's being the on-site energies for the single-level charge state in dot $i$. The Hamiltonian for SOI-free electron reservoirs, $\mathcal{H}_{\mathrm{E}}$, is described by

$$
\mathcal{H}_{\mathrm{E}}=\sum_{\alpha} \mathcal{H}_{\alpha}
$$

with

$$
\mathcal{H}_{\alpha}=\sum_{\boldsymbol{k} \in \alpha, \sigma} \epsilon_{\alpha \boldsymbol{k}} c_{\alpha \boldsymbol{k} \sigma}^{\dagger} c_{\alpha \boldsymbol{k} \sigma}
$$

where $\alpha=L, R$ labels the two leads and $\boldsymbol{k} \sigma$ denoting the states in the leads with orbital quantum number $\boldsymbol{k}$ and spin $\sigma$. Here $a_{i \sigma}^{\dagger}\left(a_{i \sigma}\right)$ and $c_{\alpha \boldsymbol{k} \sigma}^{\dagger}\left(c_{\alpha \boldsymbol{k} \sigma}\right)$ are the electron creation (annihilation) operators for electronic levels $i \sigma$ and $\boldsymbol{k} \sigma$ in the scattering area and in the lead $\alpha$, respectively. Due to the SOI, flipping of the spin can occur when an electron tunnels forth and back between the DQD and the leads. The tunneling Hamiltonian,

$$
\mathcal{H}_{\mathrm{T}}=\sum_{i \alpha \boldsymbol{k}} \sum_{\sigma \sigma^{\prime}}\left[V_{i \sigma, \alpha \boldsymbol{k} \sigma^{\prime}} a_{i \sigma}^{\dagger} c_{\alpha \boldsymbol{k} \sigma^{\prime}}+\text { h.c. }\right] \text {, }
$$

is then specified by the tunneling amplitudes,

$$
V_{i \sigma, \alpha \boldsymbol{k} \sigma^{\prime}}=V_{i \alpha \boldsymbol{k}}\left\langle\sigma\left|U^{i \alpha}\right| \sigma^{\prime}\right\rangle
$$

which contain two separate parts. The spatial part $V_{i \alpha \boldsymbol{k}}=\bar{V}_{i \alpha \boldsymbol{k}} e^{i \phi_{i \alpha}}$ embeds the $\mathrm{AB}$ phase. The phases are constrained by the relation

$$
\phi=\phi_{L}-\phi_{R}
$$

with $\phi_{\alpha}=\phi_{1 \alpha}-\phi_{2 \alpha}$ for $\alpha=L, R$. Here $\phi=\Phi / \Phi_{0}$, where $\Phi$ is the applied magnetic flux and $\Phi_{0}$ is the flux quantum. The accompanying spin rotation due to the SOI is a unitary operation $U^{i \alpha}$, determined by the underlying bonding geometry. 
Specifically, if the system lies on the $x-y$ plane, then these rotations are ${ }^{43,73}$

$$
U^{i \alpha}=\exp \left(i \mathbf{K}^{i \alpha} \cdot \boldsymbol{\sigma}\right)
$$

where $\boldsymbol{\sigma}=\sigma_{x} \hat{\mathbf{x}}+\sigma_{y} \hat{\mathbf{y}}+\sigma_{z} \hat{\mathbf{z}}$, is the vector of Pauli matrices and

$$
\begin{aligned}
\mathbf{K}^{i \alpha}= & \left(\alpha_{R} \hat{\mathbf{g}}^{i \alpha} \cdot \hat{\mathbf{y}}+\alpha_{D} \hat{\mathbf{g}}^{i \alpha} \cdot \hat{\mathbf{x}}\right) \hat{\mathbf{x}} \\
& -\left(\alpha_{R} \hat{\mathbf{g}}^{i \alpha} \cdot \hat{\mathbf{x}}+\alpha_{D} \hat{\mathbf{g}}^{i \alpha} \cdot \hat{\mathbf{y}}\right) \hat{\mathbf{y}}
\end{aligned}
$$

We denote the position of the dot $i$ by $\mathbf{r}_{i}$ and that of the connecting site on lead $\alpha$ by $\mathbf{r}_{\alpha}$. They are separated by a distance $L$. The unit vector pointing from dot $i$ to the connecting site on lead $\alpha$ is then denoted by $\hat{\mathrm{g}}^{i \alpha}=\left(\mathbf{r}_{\alpha}-\right.$ $\left.\mathbf{r}_{i}\right) / L$. In Eq. (7b), $\alpha_{R, D}=k_{R, D} L$ while $k_{R}$ and $k_{D}$ are the associated coefficients for the Rashba and (linear) Dresselhaus SOI.

It is well known that an electron acquires a phase when it moves around a loop in a region with SOI 2,73.74 In our system the two dots and the two electrodes form a loop. This SOI-induced phase is determined in the following way. Consider the unitary operators $U^{L} \equiv U^{L 1} U^{1 R} U^{R 2} U^{2 L}, U^{1} \equiv U^{1 R} U^{R 2} U^{2 L} U^{L 1}$, $U^{R} \equiv U^{R 2} U^{2 L} U^{L 1} U^{1 R}$ and $U^{2} \equiv U^{2 L} U^{L 1} U^{1 R} U^{R 2}$, where $U^{\alpha i}=\left(U^{i \alpha}\right)^{\dagger}$, which represent the rotations of the spinors related to electrons that traverse around the loop starting and ending at the sites $L, 1, R$ and 2 , respectively. The phase $\psi_{\text {so }}$ is obtained by diagonalizing these spin rotations around the loop. The results are formally given by

$$
U^{x}=e^{-i \psi_{\text {so }}}\left|\hat{\mathbf{n}}_{x} ;+\right\rangle\left\langle\hat{\mathbf{n}}_{x} ;+\left|+e^{i \psi_{\text {so }}}\right| \hat{\mathbf{n}}_{x} ;-\right\rangle\left\langle\hat{\mathbf{n}}_{x} ;-\right|,
$$

for $x=L, R, 1$, and 2 . Here $\left|\hat{\mathbf{n}}_{x} ;+\right\rangle$ and $\left|\hat{\mathbf{n}}_{x} ;-\right\rangle$ are the spinors for spin up and spin down in the direction $\hat{\mathbf{n}}_{x}$ defined via $\hat{\mathbf{n}}_{x} \cdot \boldsymbol{\sigma}\left|\hat{\mathbf{n}}_{x} ; \pm\right\rangle= \pm\left|\hat{\mathbf{n}}_{x} ; \pm\right\rangle$, where the $\hat{\mathbf{n}}_{x}$ 's are certain real unit vectors in three dimensions. The phase $\psi_{\text {so }}$ and the characteristic directions $\hat{\mathbf{n}}_{x}$ 's are fully determined from Eq. (77) and thus incorporate the full information about the SOI-induced spin rotations around the loop. The authors of Ref. [43] have shown that under the spin filter conditions (see Eq. 29) and also discussions in Ref. [43]) ) electrons come in with spinor $\left|\hat{\mathbf{n}}_{L} ; \pm\right\rangle$ from the left and will go out with spinor $\left|\hat{\mathbf{n}}_{R} ; \pm\right\rangle$ on the right (and vice versa). There the explicit dependencies of $\psi_{\text {so }}$ as well as $\hat{\mathbf{n}}_{\alpha}$ on the bonding geometry, and on the Rashba and the Dresselhaus coefficients have been discussed in detail. 75

\section{B. Correspondence to the spinless DQD AB interferometer}

\section{Decomposition into equivalent spinless systems}

Utilizing the eigenspinors of the rotations around the loop, $\left|\hat{\mathbf{n}}_{x} ; \pm\right\rangle$, in Eq. (8), the spin rotations along the sections of the loop, Eq. (7), become

$$
U^{i \alpha}=\sum_{\nu= \pm} e^{i \psi_{i \alpha}^{\nu}}\left|\mathbf{n}_{i} ; \nu\right\rangle\left\langle\mathbf{n}_{\alpha} ; \nu\right| .
$$

Here the phases $\psi_{i \alpha}^{ \pm}$'s are restrained by

$$
\pm \psi_{\mathrm{so}}=\psi_{L}^{ \pm}-\psi_{R}^{ \pm}
$$

where $\psi_{\alpha}^{ \pm}=\psi_{1 \alpha}^{ \pm}-\psi_{2 \alpha}^{ \pm}$for $\alpha=L, R$. With the aid of the basis transformation,

$$
\begin{aligned}
& a_{i \sigma}^{\dagger}=\sum_{\nu= \pm}\left\langle\hat{\mathbf{n}}_{i} ; \nu \mid \sigma\right\rangle a_{i \hat{\mathbf{n}}_{i} ; \nu}^{\dagger}, \\
& c_{\alpha \boldsymbol{k} \sigma}^{\dagger}=\sum_{\nu= \pm}\left\langle\hat{\mathbf{n}}_{\alpha} ; \nu \mid \sigma\right\rangle c_{\alpha \boldsymbol{k} \hat{\mathbf{n}}_{\alpha} ; \nu}^{\dagger},
\end{aligned}
$$

for arbitrary spinor $|\sigma\rangle$, the total Hamiltonian of the system can be decomposed into two terms,

$$
\mathcal{H}=\mathcal{H}_{+}+\mathcal{H}_{-},
$$

where,

$$
\mathcal{H}_{ \pm}=\mathcal{H}_{\mathrm{S}}^{ \pm}+\mathcal{H}_{\mathrm{E}}^{ \pm}+\mathcal{H}_{\mathrm{T}}^{ \pm}
$$

in which

$$
\begin{gathered}
\mathcal{H}_{\mathrm{S}}^{ \pm}=\sum_{i=1}^{2} E_{i} a_{i \hat{\mathbf{n}}_{i} ; \pm}^{\dagger} a_{i \hat{\mathbf{n}}_{i} ; \pm}, \\
\mathcal{H}_{\mathrm{E}}^{ \pm}=\sum_{\alpha} \mathcal{H}_{\alpha}^{ \pm}, \\
\mathcal{H}_{\alpha}^{ \pm}=\sum_{\boldsymbol{k} \in \alpha} \epsilon_{\alpha \boldsymbol{k}} c_{\alpha \boldsymbol{k} \hat{\mathbf{n}}_{\alpha} ; \pm}^{\dagger} c_{\alpha \boldsymbol{k} \hat{\mathbf{n}}_{\alpha} ; \pm},
\end{gathered}
$$

and

$$
\mathcal{H}_{\mathrm{T}}^{ \pm}=\sum_{i \alpha \boldsymbol{k}}\left[\bar{V}_{i \alpha \boldsymbol{k}} e^{i \varphi_{i \alpha}^{ \pm}} a_{i \hat{\mathbf{n}}_{i} ; \pm}^{\dagger} c_{\alpha \boldsymbol{k} \hat{\mathbf{n}}_{\alpha} ; \pm}+\text { h.c. }\right] .
$$

with

$$
\varphi_{i \alpha}^{ \pm}=\phi_{i \alpha}+\psi_{i \alpha}^{ \pm}
$$

Defining similarly $\varphi_{\alpha}^{ \pm}=\varphi_{1 \alpha}^{ \pm}-\varphi_{2 \alpha}^{ \pm}$, one directly obtains from Eqs. (6, 10, 12g) that

$$
\varphi_{ \pm} \equiv \phi \pm \psi_{\text {so }}=\varphi_{L}^{ \pm}-\varphi_{R}^{ \pm}
$$

The \pm subscript in $\varphi_{ \pm}$should not be confused with that on the operator $a_{i \hat{\mathbf{n}}_{i} ; \pm}$. The former distinguishes between the two phases in Eq. (13), while the latter denotes the spin polarization along the dot-dependent direction $\hat{\mathbf{n}}_{i}$.

The phase relation, Eq. (13), in comparison to Eq. (6), reveals that the decomposed Hamiltonian, $\mathcal{H}_{ \pm}$, Eq. (12), is the Hamiltonian for a spinless DQD AB interferometer with the flux replaced by $\varphi_{ \pm}$as the effective flux. Furthermore, by the orthogonality, $\left\langle\hat{\mathbf{n}}_{x} ; \pm \mid \hat{\mathbf{n}}_{x} ; \mp\right\rangle=0$, these two component Hamiltonians commute with each other,

$$
\left[\mathcal{H}_{+}, \mathcal{H}_{-}\right]=0 \text {. }
$$

It is therefore possible to relate the spin-resolved currents for the target system to the currents for the effective spinless setup described by $\mathcal{H}_{+}$and $\mathcal{H}_{-}$separately. 


\section{Relating the spin-resolved currents to the currents for the spinless DQD AB interferometer}

Consider an arbitrary spinor $|\hat{\mathbf{n}} ; \pm\rangle$, defined as the eigenstate of $\hat{\mathbf{n}} \cdot \boldsymbol{\sigma}$, where $\hat{\mathbf{n}}$ is an arbitrary threedimensional unit vector, by $\hat{\mathbf{n}} \cdot \boldsymbol{\sigma}|\hat{\mathbf{n}} ; \pm\rangle= \pm|\hat{\mathbf{n}} ; \pm\rangle$. Taking $|\sigma\rangle=|\hat{\mathbf{n}} ; \pm\rangle$ in Eq. (A2), the spin-resolved current on the lead $\alpha$ with the spinor $|\hat{\mathbf{n}} ; \pm\rangle$ is given by

$$
I_{\alpha \hat{\mathbf{n}} ; \pm}(t)=-\frac{d}{d t} \operatorname{tr}_{\text {tot }}\left[\mathcal{N}_{\alpha, \hat{\mathbf{n}} ; \pm} \rho_{\text {tot }}(t)\right]
$$

Setting $\hat{\mathbf{n}}=\hat{\mathbf{n}}_{\alpha}$ in Eq. (15), with the help of the property, Eq. (14), one is led to

$$
I_{\alpha \hat{\mathbf{n}}_{\alpha} ; \pm}(t)=\operatorname{tr}_{\mathrm{tot}}\left[\hat{I}_{\alpha \hat{\mathbf{n}}_{\alpha} ; \pm}(t) \rho_{\mathrm{tot}}\left(t_{0}\right)\right],
$$

where

$$
\hat{I}_{\alpha \hat{\mathbf{n}}_{\alpha} ; \pm}(t)=e^{i \mathcal{H}_{ \pm}\left(t-t_{0}\right)} \hat{I}_{\alpha \hat{\mathbf{n}}_{\alpha} ; \pm} e^{-i \mathcal{H}_{ \pm}\left(t-t_{0}\right)},
$$

is the Heisenberg representation of the current operator,

$$
\hat{I}_{\alpha \hat{\mathbf{n}}_{\alpha} ; \pm}=-i \sum_{\boldsymbol{k} \in \alpha}\left[\bar{V}_{i \alpha \boldsymbol{k}} e^{i \varphi_{i \alpha}^{ \pm}} a_{i \hat{\mathbf{n}}_{i} ; \pm}^{\dagger} c_{\alpha \boldsymbol{k} \hat{\mathbf{n}}_{\alpha} ; \pm}-\text { h.c. }\right] \text {. }
$$

On the other hand, the current on lead $\alpha$ for the spinless interferometer described by $\mathcal{H}_{ \pm}$with the effective flux $\varphi_{ \pm}$, is defined by

$$
I_{\alpha}^{0}\left(\varphi_{ \pm}, t\right)=-\frac{d}{d t} \operatorname{tr}_{\mathrm{tot}}\left[\mathcal{N}_{\alpha, \hat{\mathbf{n}}_{\alpha} ; \pm} \rho_{\mathrm{tot}}^{ \pm}(t)\right]
$$

where $\rho_{\text {tot }}^{ \pm}(t)$ is the total density matrix for the spinless system $\mathcal{H}_{ \pm}$. Similarly, Eq. (17) can be rewritten as

$$
I_{\alpha}^{0}\left(\varphi_{ \pm}, t\right)=\operatorname{tr}_{\text {tot }}\left[\hat{I}_{\alpha}^{ \pm}(t) \rho_{\text {tot }}^{ \pm}\left(t_{0}\right)\right]
$$

where

$$
\hat{I}_{\alpha}^{ \pm}(t)=e^{i \mathcal{H}_{ \pm}\left(t-t_{0}\right)} \hat{I}_{\alpha}^{ \pm} e^{-i \mathcal{H}_{ \pm}\left(t-t_{0}\right)} .
$$

The current operator in Eq. (18b) is just

$$
\hat{I}_{\alpha}^{ \pm}=\hat{I}_{\alpha \hat{\mathbf{n}}_{\alpha} ; \pm}
$$

which is given by Eq. (16c).

Here we want to study the spin polarization processes induced by the intrinsic mechanisms of SOI, without the inference of the polarization prepared in the initial states. We hence set $\rho_{\text {tot }}\left(t_{0}\right)$ to describe an unpolarized interferometer with the reservoirs in the thermal equilibrium states, namely,

$$
\rho_{\text {tot }}\left(t_{0}\right)=\rho\left(t_{0}\right) \prod_{\alpha=L, R} \rho_{\alpha}\left(t_{0}\right)
$$

where

$$
\rho_{\alpha}\left(t_{0}\right)=\frac{\exp \left[\left(\mathcal{H}_{\alpha}-\mu_{\alpha} \mathcal{N}_{\alpha}\right) / k_{B} T_{\alpha}\right]}{\operatorname{tr} \exp \left[\left(\mathcal{H}_{\alpha}-\mu_{\alpha} \mathcal{N}_{\alpha}\right) / k_{B} T_{\alpha}\right]},
$$

and $\mathcal{N}_{\alpha}=\mathcal{N}_{\alpha, \hat{\mathbf{n}} ;+}+\mathcal{N}_{\alpha, \hat{\mathbf{n}} ;-}$, for an arbitrary unit vector $\hat{\mathbf{n}}$, is the total electron number operator in lead $\alpha$. Here $\mu_{\alpha}$ and $T_{\alpha}$ are the chemical potential and the temperature for all spin species in lead $\alpha$. The initial state of the DQD does not possess any polarization and assumes the product form $\rho\left(t_{0}\right)=\rho_{\sigma} \rho_{\bar{\sigma}}$, where $\rho_{\sigma}=\rho_{0}$ describes the state of a spinless DQD, for all spins $\sigma$ and their opposite $\bar{\sigma}$. Therefore one can designate,

$$
\rho_{\text {tot }}^{ \pm}\left(t_{0}\right)=\rho_{0} \prod_{\alpha=L, R} \frac{\exp \left[\left(\mathcal{H}_{\alpha}^{ \pm}-\mu_{\alpha} \mathcal{N}_{\alpha, \hat{\mathbf{n}}_{\alpha} ; \pm}\right) / k_{B} T_{\alpha}\right]}{\operatorname{tr} \exp \left[\left(\mathcal{H}_{\alpha}^{ \pm}-\mu_{\alpha} \mathcal{N}_{\alpha, \hat{\mathbf{n}}_{\alpha} ; \pm}\right) / k_{B} T_{\alpha}\right]},
$$

to be the corresponding initial states for the effective spinless systems, such that the following identity,

$$
I_{\alpha \hat{\mathbf{n}}_{\alpha} ; \pm}(t)=I_{\alpha}^{0}\left(\varphi_{ \pm}, t\right)
$$

is held for all time $t$. The identification, Eq. (22), enables us to discuss the spin-dependent transport in the present system in terms of what has been discussed for the spinless DQD AB interferometer previously ${ }^{72}$ In the steady-state limit, where the initial preparation for the part of the DQD no longer matters, the identity Eq. (22) with $t \rightarrow \infty$ shall always be held.

The equality, Eq. (22), means that $I_{\alpha}^{0}\left(\varphi_{+}, t\right)$ and $I_{\alpha}^{0}\left(\varphi_{-}, t\right)$ respectively are the currents in lead $\alpha$ for spinup and spin-down electrons in the characteristic direction $\hat{\mathbf{n}}_{\alpha}$. Using the basis transformation, Eq. (11), with the identification, Eq. (22), the spin-resolved current on lead $\alpha$ for an arbitrary spinor, $|\hat{\mathbf{n}} ; \nu\rangle$, defined by Eq. (15), can be expressed as

$$
I_{\alpha \hat{\mathbf{n}} ; \nu}(t)=\sum_{\nu^{\prime}= \pm}\left|\left\langle\hat{\mathbf{n}}_{\alpha} ; \nu^{\prime} \mid \hat{\mathbf{n}} ; \nu\right\rangle\right|^{2} I_{\alpha}^{0}\left(\varphi_{\nu^{\prime}}, t\right) .
$$

The current formula, Eq. (23), shows that $I_{\alpha \hat{\mathbf{n}} ; \nu}(t)$ is a mixture of the currents $I_{\alpha \hat{\mathbf{n}}_{\alpha} ; \pm}(t)$ weighted by the spinor projections $\left|\left\langle\hat{\mathbf{n}}_{\alpha} ; \pm \mid \hat{\mathbf{n}} ; \nu\right\rangle\right|^{2}$. The arbitrary global phases embedded in $|\hat{\mathbf{n}} ; \nu\rangle$ and $\left|\hat{\mathbf{n}}_{\alpha} ; \pm\right\rangle$ are canceled in Eq. (23). From either Eq. (22) or Eq. (23), we find that the spinindependent total current,

$$
\begin{aligned}
I_{\alpha}(t) & \equiv I_{\alpha \hat{\mathbf{n}} ;+}(t)+I_{\alpha \hat{\mathbf{n}} ;-}(t) \\
& =I_{\alpha}^{0}\left(\varphi_{+}, t\right)+I_{\alpha}^{0}\left(\varphi_{-}, t\right),
\end{aligned}
$$

is the sum of these two currents $I_{\alpha}^{0}\left(\varphi_{+}, t\right)$ and $I_{\alpha}^{0}\left(\varphi_{-}, t\right)$. This is consistent with the analysis in Ref. [74].

\section{The rise of spin-polarized transport}

The main purpose of the present paper is to explore the dynamical rise of the spin polarization in the currents. This is intimately related to the dynamics of spin flows. The spin flow from lead $\alpha$ is

$$
\mathbf{I}_{\alpha}^{\mathrm{S}}(t)=-\frac{d}{d t} \operatorname{tr}_{\mathrm{tot}}\left[\mathbf{S}_{\alpha} \rho_{\mathrm{tot}}(t)\right],
$$


where the total spin operator for the electrode $\alpha$ (with $\hbar=1$ ) is defined by,

$$
\mathbf{S}_{\alpha}=\sum_{k \in \alpha} \sum_{\sigma \sigma^{\prime}} c_{\alpha \boldsymbol{k} \sigma}^{\dagger}\left(\frac{1}{2} \sigma\right)_{\sigma \sigma^{\prime}} c_{\alpha \boldsymbol{k} \sigma^{\prime}}
$$

Comparing Eq. (25) with Eq. (15), the spin flow from lead $\alpha$ is related to the spin-resolved currents there by

$$
\mathbf{I}_{\alpha}^{\mathrm{S}}(t)=\frac{1}{2} \sum_{i=1}^{3} \hat{\mathbf{x}}_{i}\left(I_{\alpha \hat{\mathbf{x}}_{i} ;+}(t)-I_{\alpha \hat{\mathbf{x}}_{i} ;-}(t)\right),
$$

where $\left\{\hat{\mathbf{x}}_{1}, \hat{\mathbf{x}}_{2}, \hat{\mathbf{x}}_{3}\right\}=\{\hat{\mathbf{x}}, \hat{\mathbf{y}}, \hat{\mathbf{z}}\}$. Using the identities $\left|\left\langle\hat{\mathbf{n}}^{\prime} ; \pm \mid \hat{\mathbf{n}} ; \pm\right\rangle\right|^{2}=\left(1+\hat{\mathbf{n}} \cdot \hat{\mathbf{n}}^{\prime}\right) / 2$ and $\left|\left\langle\hat{\mathbf{n}}^{\prime} ; \pm \mid \hat{\mathbf{n}} ; \mp\right\rangle\right|^{2}=$ $\left(1-\hat{\mathbf{n}} \cdot \hat{\mathbf{n}}^{\prime}\right) / 2$ for arbitrary directions $\hat{\mathbf{n}}$ and $\hat{\mathbf{n}}^{\prime}$ in Eq. (23), we can rewrite Eq. (26) as

$$
\mathbf{I}_{\alpha}^{\mathrm{S}}(t)=\frac{\hat{\mathbf{n}}_{\alpha}}{2}\left[I_{\alpha \hat{\mathbf{n}}_{\alpha} ;+}(t)-I_{\alpha \hat{\mathbf{n}}_{\alpha} ;-}(t)\right] .
$$

At the same time, Eq. (23) becomes

$$
I_{\alpha \hat{\mathbf{n}} ; \pm}(t)=\frac{I_{\alpha}(t)}{2} \pm \mathbf{I}_{\alpha}^{\mathrm{S}}(t) \cdot \hat{\mathbf{n}} .
$$

The factors behind the rise of spin-polarized transports can be read from the expression, Eq. (28). Without the SOI, $\psi_{\text {so }}=0$ and consequently $I_{\alpha}^{0}\left(\varphi_{+}, t\right)=I_{\alpha}^{0}\left(\varphi_{-}, t\right)=$ $I_{\alpha}^{0}(\phi, t)$, Eq. (28) reduces to $I_{\alpha \hat{\mathbf{n}} ;+}(t)=I_{\alpha \hat{\mathbf{n}} ;-}(t)=$ $I_{\alpha}^{0}(\phi, t)$ for arbitrary $\hat{\mathbf{n}}$. Therefore without SOI it is not possible to have spin flow in this system, as expected. Only when SOI is present, the effective fluxes $\varphi_{+}$and $\varphi_{-}$can be different. The expression, Eq. (28), together with Eq. (22), manifests that because spin-up electrons and spin-down electrons experience different effective fluxes $\varphi_{+}$and $\varphi_{-}$, it is possible to have $\mathbf{I}_{\alpha}^{\mathrm{S}}(t) \neq 0$. This underlies the occurrence of a preferred spin direction in the currents. Note that in the steady-state limit, $t \rightarrow \infty$, the two-terminal spinless interferometers are subjected to phase rigidity, $I_{\alpha}^{0}(\varphi)=I_{\alpha}^{0}(-\varphi)$. If there is no applied flux, $\phi=0$, then $\varphi_{ \pm}= \pm \psi_{\text {so }}$ and therefore $I_{\alpha}^{0}\left(\varphi_{+}\right)=I_{\alpha}^{0}\left(\varphi_{-}\right)$and $\mathbf{I}_{\alpha}^{\mathrm{S}}=0$. This demonstrates the importance of the combined effect of the flux and the SOI for maintaining spin polarization in the currents to the steady-state limit. We will give also explicit calculations showing this result in later sections.

A very important consequence of Eq. (28) is that whenever there is a non-vanishing spin current $\mathbf{I}_{\alpha}^{\mathrm{S}}(t) \neq 0$, the current on lead $\alpha$ is always polarized in the characteristic direction $\hat{\mathbf{n}}_{\alpha}$ for all time $t$, which is fixed by the SOI parameters of the system. Henceforth, to obtain a fully spin-polarized current, one requires either $I_{\alpha \hat{\mathbf{n}}_{\alpha} ;+}(t)$ or $I_{\alpha \hat{\mathbf{n}}_{\alpha} ;-}(t)$ to vanish. The relation of Eq. (22) indicates that such a task could be fulfilled by making one of the currents for the effective spinless systems, $I_{\alpha}^{0}\left(\varphi_{+}, t\right)$ or $I_{\alpha}^{0}\left(\varphi_{-}, t\right)$, diminish while the other remains finite. Note that since generally $\hat{\mathbf{n}}_{L} \neq \hat{\mathbf{n}}_{R}$, the current on the left and that on the right are polarized along different directions.
It is pointed out in Ref. [43] that such a system can give rise to full spin polarization when two conditions are fulfilled. The first condition is that the upper arm and the lower arm of the interferometer are symmetrically set up, namely,

$$
\begin{aligned}
& \bar{V}_{1 \alpha k}=\bar{V}_{2 \alpha k}=\bar{V}_{\alpha k}, \\
& E_{1}=E_{2}=E .
\end{aligned}
$$

The second condition is that the applied magnetic flux and the underlying SOI parameters should be chosen to satisfy

$$
\cos \left(\varphi_{-}\right)=-1 .
$$

These conditions were obtained from a scattering analysis with a tight-binding modeling of the two leads. Indeed, applying these conditions to the total Hamiltonian of the target system, we confirm that their validity is independent of the energy dispersion in the leads. We also find that the rise of the fully spin-polarized transport is equivalent to a completely destructive interference in the corresponding spinless interferometer, described by $\mathcal{H}_{-}$. Such effects can be seen by analyzing the component Hamiltonians $\mathcal{H}_{ \pm}$in the decomposition, Eq. (12).

To highlight the role played by the effective flux, we perform a gauge transformation to the Hamiltonians $\mathcal{H}_{ \pm}$, yielding

$$
\begin{gathered}
\mathcal{H}_{\mathrm{S}}^{ \pm}=\sum_{i=1}^{2} E_{i} d_{i \hat{\mathbf{n}}_{i} ; \pm}^{\dagger} d_{i \hat{\mathbf{n}}_{i} ; \pm}, \\
\mathcal{H}_{\mathrm{E}}^{ \pm}=\sum_{\alpha \boldsymbol{k}} \epsilon_{\alpha \boldsymbol{k}} b_{\alpha \boldsymbol{k} \hat{\mathbf{n}}_{\alpha} ; \pm}^{\dagger} b_{\alpha \boldsymbol{k} \hat{\mathbf{n}}_{\alpha} ; \pm},
\end{gathered}
$$

and

$$
\begin{aligned}
& \mathcal{H}_{\mathrm{T}}^{ \pm}= \\
& \sum_{\boldsymbol{k}}\left[\left(\overline{\mathcal{V}}_{1 L \boldsymbol{k}} e^{i \varphi_{ \pm} / 4} d_{1 \hat{\mathbf{n}}_{1} ; \pm}^{\dagger}+\overline{\mathcal{V}}_{2 L \boldsymbol{k}} e^{-i \varphi_{ \pm} / 4} d_{2 \hat{\mathbf{n}}_{2} ; \pm}^{\dagger}\right) b_{L \boldsymbol{k} \hat{\mathbf{n}}_{\alpha} ; \pm}\right. \\
& \left.+\left(\overline{\mathcal{V}}_{1 R \boldsymbol{k}} e^{-i \varphi_{ \pm} / 4} d_{1 \hat{\mathbf{n}}_{1 ;} ; \pm}^{\dagger}+\overline{\mathcal{V}}_{2 R \boldsymbol{k}} e^{i \varphi_{ \pm} / 4} d_{2 \hat{\mathbf{n}}_{2} ; \pm}^{\dagger}\right) b_{R \boldsymbol{k} \hat{\mathbf{n}}_{R} ; \pm}\right] \\
& + \text { h.c. }
\end{aligned}
$$

where the newly defined operators and amplitudes are

$$
\begin{aligned}
& d_{1 \hat{\mathbf{n}}_{1} ; \pm}^{\dagger}=e^{i \chi_{ \pm} / 2} a_{1 \hat{\mathbf{n}}_{1} ; \pm}^{\dagger}, \quad d_{2 \hat{\mathbf{n}}_{2} ; \pm}^{\dagger}=e^{-i \chi_{ \pm} / 2} a_{2 \hat{\mathbf{n}}_{2} ; \pm}^{\dagger}, \\
& b_{L \boldsymbol{k} \hat{\mathbf{n}}_{L} ; \pm}=e^{i \delta \theta_{ \pm}} c_{L \boldsymbol{k} \hat{\mathbf{n}}_{L} ; \pm}, \quad b_{R \boldsymbol{k} \hat{\mathbf{n}}_{R} ; \pm}=e^{-i \delta \theta_{ \pm}} c_{R \boldsymbol{k} \hat{\mathbf{n}}_{R} ; \pm}
\end{aligned}
$$

and

$$
\overline{\mathcal{V}}_{i \alpha \boldsymbol{k}}=\bar{V}_{i \alpha \boldsymbol{k}} e^{i \bar{\theta}_{ \pm}},
$$

in which the free gauges are

$$
\begin{aligned}
& \chi_{ \pm}=\left(\varphi_{L}^{ \pm}+\varphi_{R}^{ \pm}\right) / 2, \\
& \delta \theta_{ \pm}=\left(\theta_{L}-\theta_{R}\right) / 4, \\
& \bar{\theta}_{ \pm}=\left(\theta_{L}+\theta_{R}\right) / 4,
\end{aligned}
$$


with $\theta_{\alpha}=\varphi_{1 \alpha}^{ \pm}+\varphi_{2 \alpha}^{ \pm}$.

Applying the condition, Eq. (29a), the tunneling parts in the Hamiltonians $\mathcal{H}_{+}$and $\mathcal{H}_{-}$, can be written as,

$$
\mathcal{H}_{\mathrm{T}}^{ \pm}=\mathcal{H}_{\mathrm{T}}^{L \pm}+\mathcal{H}_{\mathrm{T}}^{R \pm}
$$

where

$$
\mathcal{H}_{\mathrm{T}}^{\alpha \pm}=\left[\sum_{\boldsymbol{k} \in \alpha} \overline{\mathcal{V}}_{\alpha \boldsymbol{k}} \tilde{d}_{\alpha \pm}^{\dagger} b_{\alpha \boldsymbol{k} \hat{\mathbf{n}}_{\alpha} ; \pm}+\text { h.c. }\right] \sqrt{2}
$$

with

$$
\begin{aligned}
& \tilde{d}_{L \pm}^{\dagger}=\left(e^{i \varphi_{ \pm} / 4} d_{1 \hat{\mathbf{n}}_{1} ; \pm}^{\dagger}+e^{-i \varphi_{ \pm} / 4} d_{2 \hat{\mathbf{n}}_{2} ; \pm}^{\dagger}\right) / \sqrt{2}, \\
& \tilde{d}_{R \pm}^{\dagger}=\left(e^{-i \varphi_{ \pm} / 4} d_{1 \hat{\mathbf{n}}_{1} ; \pm}^{\dagger}+e^{+i \varphi_{ \pm} / 4} d_{2 \hat{\mathbf{n}}_{2} ; \pm}^{\dagger}\right) / \sqrt{2},
\end{aligned}
$$

where the factor $1 / \sqrt{2}$ is for normalization. This shows that for $\mathcal{H}_{ \pm}$the left and the right electrodes respectively couple to the modes $|L \pm\rangle=\tilde{d}_{L \pm}^{\dagger}|0\rangle$ and $|R \pm\rangle=\tilde{d}_{R \pm}^{\dagger}|0\rangle$, where $|0\rangle$ denotes the empty state of the DQD. The overlap between them is

$$
\langle L \pm \mid R \pm\rangle=\sqrt{\frac{\cos \left(\varphi_{ \pm}\right)+1}{2}} .
$$

When $\varphi_{-}$satisfies Eq. (29c), these two modes become orthogonal. By further setting the on-site energies of the DQD to be degenerate, Eq. (29b), the effective spinless system described by $\mathcal{H}_{-}$is actually split into two separate systems, each of which is a single-level dot coupled to a reservoir (see Fig. 2(a)). The current on lead $\alpha$, $I_{\alpha}^{0}\left(\varphi_{-}, t\right)$, in this disconnected setup will eventually reach zero. This picture of disconnected electrodes underlies the completely destructive interference for the spinless interferometer. This effect in turn gives the vanishing steady-state current,

$$
I_{\alpha \hat{\mathbf{n}}_{\alpha} ;-}(t \rightarrow \infty)=0,
$$

for the spinor $\left|\hat{\mathbf{n}}_{\alpha} ;-\right\rangle$ in lead $\alpha$.

By the same token, the effective configuration for the connection between the two reservoirs for $\mathcal{H}_{+}$is controlled by the value of $\varphi_{+}$. As long as $\varphi_{+}$does not satisfy $\cos \left(\varphi_{+}\right)=-1$, the two electrodes for $\mathcal{H}_{+}$stay connected, supporting a non-vanishing current,

$$
I_{\alpha \hat{\mathbf{n}}_{\alpha} ;+}(t \rightarrow \infty) \neq 0,
$$

provided that a nonzero bias is applied. Noticeably, when $\varphi_{+}=2 m \pi$ with $m$ being an arbitrary integer, then the overlap between the two modes, $|L+\rangle$ and $|R+\rangle$ becomes unity. This means that, at degeneracy, the transport only goes through one mode, which is a linear combination of the original two QD's charge states of equal weights. This opposite limit is contrasted in Fig. 2(b).

The difference between the effective configurations for $\mathcal{H}_{-}$and $\mathcal{H}_{+}$has led to the different dependencies of the dynamical evolutions of the currents $I_{\alpha \hat{\mathbf{n}}_{\alpha} ;-}(t)$ and $I_{\alpha \hat{\mathbf{n}}_{\alpha} ;+}(t)$ on the target system's parameters. The configuration of Fig. 2(a) implies that the current carrying the characteristic spinor $\left|\hat{\mathbf{n}}_{\alpha} ;-\right\rangle$ in lead $\alpha$ is only affected by the parameters concerning the reservoir $\alpha$ and its coupling to the DQD, whereas the opposite reservoir $\bar{\alpha}$ exerts no influence. On the contrary, for $\mathcal{H}_{+}$, the connected configuration asserts that $I_{\alpha \hat{\mathbf{n}}_{\alpha} ;+}(t)$ is affected by couplings to both of the reservoirs and their respective structures, Fig. 2(b). Explicit calculations of these spin-resolved currents demonstrating such effects will be given in later sections.

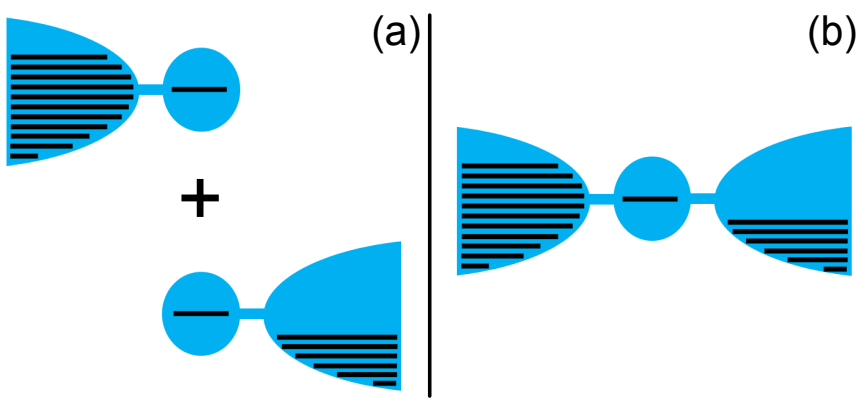

FIG. 2: (color online) Illustration of the effective spinless configuration mapped from the case of having fully polarized transport current. (a) Two separate systems, each of which is a single-level dot coupled to a reservoir. (b) a single-level dot coupled to two reservoirs.

\section{Real-time spin-dependent currents in terms of the Green functions}

In order to investigate the current polarization dynamics purely induced by the SOI, we let the central area initially contain no excess electrons. As we showed in Sec. II B and Sec. II C, the spin-dependent currents in the target system can be discussed in terms of the currents of the corresponding spinless systems with effective fluxes. The transient dynamics of these spinless interferometers have been studied before ${ }^{71,72}$ To make comparisons with our previous results easier and also to facilitate the readers familiar with standard NEGF, $, 57,77$ the notations of Keldysh NEGF are translated to those used in Ref. 72. as

$$
\begin{aligned}
& \boldsymbol{\Sigma}_{\alpha}^{r}(t, \tau)=-i \boldsymbol{g}_{\alpha}(t-\tau), \\
& \boldsymbol{\Sigma}_{\alpha}^{<}(t, \tau)=i \widetilde{\boldsymbol{g}}_{\alpha}(t-\tau),
\end{aligned}
$$

for the self-energies and

$$
\begin{aligned}
\mathbf{G}^{r}\left(\tau, t_{0}\right) & =-i \boldsymbol{u}(\tau), \\
\mathbf{G}^{a}(\tau, t) & =i \overline{\boldsymbol{u}}(\tau), \\
\mathbf{G}^{<}(\tau, t) & =i \boldsymbol{v}(\tau),
\end{aligned}
$$

for the Green functions ${ }^{67}$ The spin-resolved real-time current in terms of Keldysh NEGF is summarized in Appendix $\mathrm{A}$. 
The current for the effective spinless interferometer reads $\frac{72}{2}$

$$
\begin{aligned}
& I_{\alpha}^{0}\left(\varphi_{ \pm}, t\right) \\
& =2 \operatorname{Re} \operatorname{Tr} \int_{0}^{t} d \tau\left(\tilde{\mathbf{g}}_{\alpha}^{ \pm}(t-\tau) \overline{\mathbf{u}}^{ \pm}(\tau)-\mathbf{g}_{\alpha}^{ \pm}(t-\tau) \mathbf{v}^{ \pm}(\tau)\right) .
\end{aligned}
$$

The Green functions for the effective spinless system satisfy the equations,

$$
\partial_{\tau} \mathbf{u}^{ \pm}(\tau)+i \mathbf{E} \mathbf{u}^{ \pm}(\tau)+\int_{t_{0}}^{\tau} d \tau^{\prime} \mathbf{g}^{ \pm}\left(\tau-\tau^{\prime}\right) \mathbf{u}^{ \pm}\left(\tau^{\prime}\right)=0
$$

and

$$
\mathbf{v}^{ \pm}(\tau)=\int_{t_{0}}^{\tau} d \tau_{1} \int_{t_{0}}^{t} d \tau_{2} \mathbf{u}^{ \pm}\left(\tau-\tau_{1}\right) \tilde{\mathbf{g}}^{ \pm}\left(\tau_{1}-\tau_{2}\right) \overline{\mathbf{u}}^{ \pm}\left(\tau_{2}\right),
$$

with $\overline{\mathbf{u}}^{ \pm}(\tau)=\mathbf{u}^{ \pm}\left(t-\tau+t_{0}\right)^{\dagger}$ and the boundary condition $\mathbf{u}^{ \pm}(0)=\mathbf{1}$ is imposed. The self-energies for the effective spinless system are $\mathbf{g}^{ \pm}(\tau)=\sum_{\alpha} \mathbf{g}_{\alpha}^{ \pm}(\tau)$ and $\tilde{\mathbf{g}}^{ \pm}(\tau)=\sum_{\alpha} \tilde{\mathbf{g}}_{\alpha}^{ \pm}(\tau)$ with

$$
\begin{aligned}
& {\left[\mathbf{g}_{\alpha}^{ \pm}(\tau)\right]_{i j}=\left[\overline{\mathbf{g}}_{\alpha}(\tau)\right]_{i j} e^{i\left(\varphi_{i \alpha}^{ \pm}-\varphi_{j \alpha}^{ \pm}\right)},} \\
& {\left[\tilde{\mathbf{g}}_{\alpha}^{ \pm}(\tau)\right]_{i j}=\left[\tilde{\overline{\mathbf{g}}}_{\alpha}(\tau)\right]_{i j} e^{i\left(\varphi_{i \alpha}^{ \pm}-\varphi_{j \alpha}^{ \pm}\right)},}
\end{aligned}
$$

and

$$
\begin{aligned}
\overline{\mathbf{g}}_{\alpha}(\tau) & =\int \frac{d \omega}{2 \pi} \overline{\boldsymbol{\Gamma}}^{\alpha}(\omega) e^{-i \omega \tau}, \\
\tilde{\mathbf{g}}_{\alpha}(\tau) & =\int_{-\infty}^{\infty} \frac{d \omega}{2 \pi} f_{\alpha}(\omega) \overline{\boldsymbol{\Gamma}}^{\alpha}(\omega) e^{-i \omega \tau},
\end{aligned}
$$

in which

$$
\left[\overline{\boldsymbol{\Gamma}}^{\alpha}(\omega)\right]_{i j}=2 \pi \sum_{k \in \alpha} \bar{V}_{i \alpha k} \bar{V}_{j \alpha k}^{*} \delta\left(\omega-\varepsilon_{\alpha k}\right) .
$$

Here $\mathbf{E}=\left(\begin{array}{cc}E_{1} & 0 \\ 0 & E_{2}\end{array}\right)$ is the on-site energy matrix for the DQD. Explicitly, the matrices of the effective selfenergies are,

$$
\mathbf{g}^{ \pm}(\tau)=\left(\begin{array}{cc}
{\left[\overline{\mathbf{g}}_{L}(\tau)\right]_{11}+\left[\overline{\mathbf{g}}_{R}(\tau)\right]_{11}} & e^{i \chi_{ \pm}}\left(\left[\overline{\mathbf{g}}_{L}(\tau)\right]_{12} e^{i \varphi_{ \pm} / 2}+\left[\overline{\mathbf{g}}_{R}(\tau)\right]_{12} e^{-i \varphi_{ \pm} / 2}\right) \\
e^{-i \chi_{ \pm}}\left(\left[\overline{\mathbf{g}}_{L}(\tau)\right]_{21} e^{-i \varphi_{ \pm} / 2}+\left[\overline{\mathbf{g}}_{R}(\tau)\right]_{21} e^{i \varphi_{ \pm} / 2}\right) & {\left[\overline{\mathbf{g}}_{L}(\tau)\right]_{22}+\left[\overline{\mathbf{g}}_{R}(\tau)\right]_{22}}
\end{array}\right)
$$

where $\chi_{ \pm}$is an arbitrary gauge phase given in Eq. (31c). Straightforwardly, solving Eq. (38a) by Laplace transformation and substituting the solution into Eq. (37), we found the current, as a physical observable, is independent of the arbitrary gauge phase $\chi_{ \pm}$that appears in Eq. (40). By taking $\hat{\mathbf{n}}=\hat{\mathbf{n}}_{\alpha}$ in Eq. (23), together with Eq. (37), one immediately verifies Eq. (22).

The Green functions, Eq. (38), together with the current expression, Eq. (37), and the identity, Eq. (22), form the basis of exploring the spin-dependent electron transport described by Eq. (28). The self-energies $\mathbf{g}^{ \pm}(\tau)$, $\tilde{\mathbf{g}}^{ \pm}(\tau)$ and the Green functions $\mathbf{u}^{ \pm}(\tau), \mathbf{v}^{ \pm}(\tau)$ are respectively $\boldsymbol{g}(\tau), \tilde{\boldsymbol{g}}(\tau)$ and $\boldsymbol{u}(\tau), \boldsymbol{v}(\tau)$ found through the replacement of $\phi$ in Ref. [72] by $\varphi_{ \pm}$.

For explicit calculations, we take the commonly assumed wide-band limit. The effective self-energy functions, Eq.(40), become

$$
\mathbf{g}^{ \pm}(\tau)=\delta(\tau)\left(\begin{array}{cc}
\Gamma & e^{i \chi \pm} m_{ \pm} \\
e^{-i \chi_{ \pm}} m_{ \pm}^{*} & \Gamma
\end{array}\right)
$$

where $m_{ \pm}=\left(\Gamma_{L} e^{i \varphi_{ \pm} / 2}+\Gamma_{R} e^{-i \varphi_{ \pm} / 2}\right)$. The broadening due to the coupling to electrode $\alpha$ is $\Gamma_{\alpha}$ and $\Gamma=\Gamma_{L}+\Gamma_{R}$. The explicit expressions of the Green functions as well as the spinless currents under the wide-band assumption can be found in Ref. 72.

\section{SPIN-DEPENDENT CURRENT IN THE STEADY STATE}

Before we proceed to investigate the dynamical processes, we first take the steady-state limit. We examine the conditions for generating spin-polarized current. We reproduce known results about spin polarization. We also discuss other possibilities of spin dependence of the steady-state currents. In the end of this section, we investigate how to extract the spin-polarized transmission from the spin-independent total transmission at various electric and magnetic fields.

\section{A. Spin-polarized currents in the steady state}

The spin-resolved current, $I_{\alpha \hat{\mathbf{n}} ; \pm}(t)$, along an arbitrary direction, $\mathbf{n}$, involves both the currents $I_{\alpha}^{0}\left(\varphi_{+}, t\right)$ and $I_{\alpha}^{0}\left(\varphi_{-}, t\right)$. As seen from Ref. [72], each of these currents may require a different time to approach its steady state, determined from the rates,

$$
\gamma_{\varphi_{ \pm}}^{ \pm}=\frac{1}{2}\left(\Gamma \pm \Gamma_{\varphi_{ \pm}}\right)
$$


depending on the effective flux, $\varphi_{ \pm}$, through

$$
\Gamma_{\varphi_{ \pm}}=\sqrt{\Gamma_{L}^{2}+\Gamma_{R}^{2}+2 \Gamma_{L} \Gamma_{R} \cos \left(\varphi_{ \pm}\right)-\delta E^{2}},
$$

where $\delta E=E_{1}-E_{2}$. When $\gamma_{\varphi_{+}}^{-}$is nonzero, the time for $I_{\alpha}^{0}\left(\varphi_{ \pm}, t\right)$ to reach its steady state must be larger than $1 / \operatorname{Re}\left[\gamma_{\varphi_{ \pm}}^{-}\right]$. When $\delta E=0$ and the effective flux $\varphi_{ \pm}$is an even multiple of $\pi$, then $\gamma_{\varphi_{ \pm}}^{-}=0$ and $\gamma_{\varphi_{ \pm}}^{+}=\Gamma$. In this

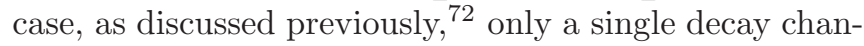
nel is present and the corresponding decay rate is just $\Gamma$. The time to reach the steady state in this situation must be lager than $1 / \Gamma$.

Taking the steady-state limit of Eq. (28), $I_{\alpha \hat{\mathbf{n}} ; \pm}(t \rightarrow$ $\infty)=I_{\alpha \hat{\mathbf{n}} ; \pm}^{\infty}$, the current from the lead $\alpha$ carrying spinor $|\hat{\mathbf{n}} ; \pm\rangle$ becomes

$$
I_{\alpha \hat{\mathbf{n}} \pm}^{\infty}=\frac{1}{2}\left[\left(1 \pm \hat{\mathbf{n}}_{\alpha} \cdot \hat{\mathbf{n}}\right) I_{\alpha}^{0}\left(\varphi_{+}\right)+\left(1 \mp \hat{\mathbf{n}}_{\alpha} \cdot \hat{\mathbf{n}}\right) I_{\alpha}^{0}\left(\varphi_{-}\right)\right]
$$

where

$$
I_{\alpha}^{0}\left(\varphi_{ \pm}\right)=\int \frac{d \omega}{2 \pi}\left(f_{\alpha}(\omega)-f_{\bar{\alpha}}(\omega)\right) T^{0}\left(\varphi_{ \pm}, \omega\right),
$$

in which $\bar{\alpha}=R$ if $\alpha=L$ and vice versa. Here the linear response of the effective spinless system with effective flux $\varphi_{ \pm}$is given by the transmission,

$T^{0}\left(\varphi_{ \pm}, \omega\right)=4 \Gamma_{L} \Gamma_{R} \frac{\omega^{2} \cos ^{2}\left(\varphi_{ \pm} / 2\right)+\left(\frac{\delta E}{2} \sin \left(\varphi_{ \pm} / 2\right)\right)^{2}}{\left(\omega^{2}+\left(\gamma_{\varphi_{ \pm}}^{+}\right)^{2}\right)\left(\omega^{2}+\left(\gamma_{\varphi_{ \pm}}^{-}\right)^{2}\right)}$.

Due to charge conservation, the steady-state currents for the spinless DQD AB interferometer are subjected to

$$
I_{L}^{0}\left(\varphi_{ \pm}\right)=-I_{R}^{0}\left(\varphi_{ \pm}\right) .
$$

Using Eq. (22), we immediately find that

$$
I_{L \hat{\mathbf{n}}_{L} \pm}^{\infty}=-I_{R \hat{\mathbf{n}}_{R} \pm}^{\infty} \cdot
$$

This indicates that the current for spinor $\left|\hat{\mathbf{n}}_{L} ; \pm\right\rangle$ leaving the left side is converted to the current for $\left|\hat{\mathbf{n}}_{R} ; \pm\right\rangle$ in the right side. It also reveals the effects of SOI when electrons are transferred across the DQD from one lead to the other. Directly from Eq. (45) or more generally from Eq. (43), we have the total current conversation,

$$
I_{L}=-I_{R}
$$

Applying the conditions for realizing the full spinpolarization in Eq. (29) to Eq. (43) results in

$$
\begin{gathered}
I_{\alpha \hat{\mathbf{n}}_{\alpha} ;-}^{\infty}=0, \\
I_{\alpha \hat{\mathbf{n}}_{\alpha} ;+}^{\infty} \neq 0 .
\end{gathered}
$$

Therefore the current on lead $\alpha$ is polarized to carry only the spinor $\left|\hat{\mathbf{n}}_{\alpha} ;+\right\rangle$ while that for the opposite spinor $\left|\hat{\mathbf{n}}_{\alpha} ;-\right\rangle$ vanishes. Equations (47), obtained in the wideband limit, are the same as Eqs. (33/34), deduced independently of the form of the level-broadening function given in Sec. IIC Our results here thus reproduce the findings in Ref. [43].

Given that the two conditions for full polarization are satisfied, the polarized current on lead $\alpha$ is described by

$$
\begin{array}{r}
I_{\alpha \hat{\mathbf{n}}_{\alpha} ;+}^{\infty}=4 \Gamma_{L} \Gamma_{R} \int \frac{d \omega}{2 \pi}\left(f_{\alpha}(\omega)-f_{\bar{\alpha}}(\omega)\right) \\
\times \frac{\omega^{2} \cos ^{2}\left(\varphi_{+} / 2\right)}{\left(\omega^{2}+\left(\gamma_{\varphi_{+}}^{+}\right)^{2}\right)\left(\omega^{2}+\left(\gamma_{\varphi_{+}}\right)^{2}\right)} .
\end{array}
$$

Setting $\mu_{L}=-\mu_{R}=e V / 2$ at zero temperature, the integral in the above equation can be done explicitly, yielding

$$
\begin{aligned}
& I_{\alpha \hat{\mathbf{n}}_{\alpha} ;+}^{\infty}= \pm \frac{4 \Gamma_{L} \Gamma_{R}}{\pi \Gamma \Gamma_{\varphi}} \cos ^{2}\left(\varphi_{+} / 2\right) \\
& \times\left[\gamma_{\varphi_{+}}^{+} \tan ^{-1}\left(\frac{e V}{2 \gamma_{\varphi_{+}}^{+}}\right)-\gamma_{\varphi_{+}}^{-} \tan ^{-1}\left(\frac{e V}{2 \gamma_{\varphi_{+}}^{-}}\right)\right],
\end{aligned}
$$

where the overall sign \pm on the right-hand side takes + for $\alpha=L$ and - for $\alpha=R$. This shows that the magnitude of the fully polarized currents sensitively depends on the effective flux $\varphi_{+}$mainly through the term $\cos ^{2}\left(\varphi_{+} / 2\right)$.

Opposite to the fully spin-polarized current is the randomly polarized current, namely, $I_{\alpha \hat{\mathbf{n}} ;+}=I_{\alpha \hat{\mathbf{n}} ;-}$, for any direction $\hat{\mathbf{n}}$. There are two possibilities for such unpolarized transport to occur. The first is the trivial situation where the SOI is switched off. The currents remain unpolarized not only in the steady-state limit but also throughout the time, as shown in the previous discussion below Eq. (28). The second circumstance is that there is no applied magnetic flux, $\phi=0$. In this case, we have $\varphi_{ \pm}= \pm \psi_{\text {so }}$ and $I_{\alpha}^{0}\left(\psi_{\text {so }}\right)=I_{\alpha}^{0}\left(-\psi_{\text {so }}\right)$ due to phase rigidity because the effective spinless interferometer is a twoterminal system [see also Eqs. 43b 43c] ]. Putting this into Eq. (43a), one immediately obtains $I_{\alpha \hat{\mathbf{n}} ;+}=I_{\alpha \hat{\mathbf{n}} ;-}$, for all directions $\hat{\mathbf{n}}$. This result exemplifies the discussion about the steady-state limit below Eq. (28) in Sec. IIC The currents in the steady-state limit are unpolarized but they may be transiently polarized (see the later discussion in Sec. IVB . When one is away from either of the two conditions for full spin polarization, one cannot reach $T^{0}\left(\varphi_{-}, \omega\right)=0$ for all $\omega$. Therefore in general both $I_{\alpha \hat{\mathbf{n}}_{\alpha}-}$ and $I_{\alpha \hat{\mathbf{n}}_{\alpha}+}$ are nonzero and the currents are only partially polarized.

\section{B. Extracting spin-resolved transmission from measurement of total transmission}

In the absence of the magnetic flux, the line shape of the spin-independent total transmission depends solely on the SOI-induced phase. Therefore one can extract 
$\psi_{\text {so }}$ from the total transmission. This in turn can be used to map out the relations between the values of the transmission for the underlying spinless system and the effective fluxes, $T^{0}(\varphi, \omega)$.

The spin-independent total charge transport current is defined by

$$
I=\frac{1}{2}\left(I_{L}-I_{R}\right)
$$

Using Eq. (43) with Eq. (24), the charge transport current, Eq. (50), in the steady-state limit becomes

$$
I=\int \frac{d \omega}{2 \pi}\left(f_{L}(\omega)-f_{R}(\omega)\right) T\left(\phi, \psi_{\mathrm{so}}, \omega\right),
$$

where the total charge transmission, $T\left(\phi, \psi_{\mathrm{so}}, \omega\right)$, is

$$
T\left(\phi, \psi_{\mathrm{so}}, \omega\right)=T^{0}\left(\varphi_{+}, \omega\right)+T^{0}\left(\varphi_{-}, \omega\right) .
$$

The zero bias charge conductance at low temperature, as the linear response, is simply given by $T\left(\phi, \psi_{\text {so }}, \omega\right)$ in Eq. (51). By turning off the magnetic flux, the total charge transmission becomes

$$
T\left(\phi=0, \psi_{\mathrm{so}}, \omega\right)=2 T^{0}\left(\psi_{\mathrm{so}}, \omega\right)
$$

where we have utilized $T^{0}\left(\psi_{\mathrm{so}}, \omega\right)=T^{0}\left(-\psi_{\mathrm{so}}, \omega\right)$.

We discuss separately the cases with $\delta E=0$ and with $\delta E \neq 0$. By tuning the on-site energies such that $\delta E=0$, with $\psi_{\text {so }}=2 n \pi$, where $n$ is an arbitrary integer, Eq. 433c) becomes a Lorentzian line shape,

$$
T^{0}(2 n \pi, \omega)=\frac{4 \Gamma_{L} \Gamma_{R}}{\left(\omega^{2}+\Gamma^{2}\right)},
$$

as shown by the most front plot in the left panel of Fig. 3. For $\psi_{\text {so }} \neq 2 n \pi$, the single-peak profile splits into two peaks, as shown by the other plots in the left panel of Fig. 3. The separation of the two peaks is given by

$$
\Delta \omega=\sqrt{2 \Gamma_{L} \Gamma_{R}\left(1-\cos \left(\psi_{\mathrm{so}}\right)\right)} .
$$

For $\psi_{\text {so }}=(2 n-1) \pi$, the transmissions vanish, as shown by the most rear plot on the left panel of Fig. 3 .

With $\delta E \neq 0, T^{0}\left(\psi_{\text {so }}, \omega\right)$ for $\psi_{\text {so }}=2 n \pi$ shows split peaks and its value at $\omega=0$ equals to zero. This is the most front plot on the right panel of Fig. 3. The separation between the two peaks in this case becomes

$$
\Delta \omega=\sqrt{2}|\delta E|
$$

For $\psi_{\text {so }} \neq 2 n \pi$, the line shape of the transmission may exhibit two peaks or a single peak profile, depending on the relations between $\psi_{\text {so }}$ and the nonzero value of $\delta E$. From Eq. (43c), we find that if

$$
\begin{aligned}
\bar{\gamma}\left(\psi_{\mathrm{so}}\right) & \equiv 4\left(\gamma_{\psi_{\mathrm{so}}}^{+} \gamma_{\psi_{\mathrm{so}}}^{-}\right)^{2} \cos ^{2}\left(\psi_{\mathrm{so}} / 2\right) \\
& -\left[\left(\gamma_{\psi_{\mathrm{so}}}^{+}\right)^{2}+\left(\gamma_{\psi_{\mathrm{so}}}^{-}\right)^{2}\right] \delta E^{2} \sin ^{2}\left(\psi_{\mathrm{so}} / 2\right)>0
\end{aligned}
$$

is satisfied, then there emerges a profile with two peaks, as exemplified by the second most front plot on the right panel of Fig. 3. The separation between these two peaks is given by

$$
\begin{aligned}
\Delta \omega & =\left\{\frac{-\frac{\delta E^{2}}{2} \sin ^{2}\left(\psi_{\mathrm{so}} / 2\right)}{2 \cos ^{2}\left(\psi_{\mathrm{so}} / 2\right)}+\right. \\
& \left.\frac{\sqrt{\left[\frac{\delta E^{2}}{2} \sin ^{2}\left(\psi_{\mathrm{so}} / 2\right)\right]^{2}+\bar{\gamma}\left(\psi_{\mathrm{so}}\right) \cos ^{2}\left(\psi_{\mathrm{so}} / 2\right)}}{2 \cos ^{2}\left(\psi_{\mathrm{so}} / 2\right)}\right\}^{1 / 2} .
\end{aligned}
$$

When the condition, Eq. (56), is not fulfilled, the transmission line shape has a single peak, but it is not a Lorentzian profile (see the plots on the right panel behind the second most front one in Fig. 3). The single peak occurs at $\omega=0$ with the height

$$
T^{0}\left(\psi_{\text {so }} \neq 2 n \pi, \omega=0\right)=\frac{16 \Gamma_{L} \Gamma_{R} \delta E^{2} \sin ^{2}\left(\psi_{\mathrm{so}} / 2\right)}{\left[\delta E^{2}+4 \Gamma_{L} \Gamma_{R} \sin ^{2}\left(\psi_{\mathrm{so}} / 2\right)\right]^{2}} .
$$

The height of the peak depends on $\delta E$ and on $\psi_{\text {so. In }}$ particular, when $\delta E=0$ then Eq. (53) yields the height $4 \Gamma_{L} \Gamma_{R} / \Gamma^{2}$.

Therefore by observing the zero bias conductance profile at given electric field without applying the magnetic flux, one can extract the SOI-induced phase $\psi_{\text {so }}$ from Eqs. (54 58) at that electric field. The other way around, one can also fix the electric field at which $\psi_{\text {so }}=2 n \pi$, and find out the value of $\phi$ at a given magnetic field through a similar procedure by the property, $T\left(\phi, \psi_{\text {so }}=2 n \pi, \omega\right)=2 T^{0}(\phi, \omega)$. Using these results, one can map out the dependence of $T^{0}(\varphi, \omega)$ on the effective flux $\varphi$. Together with the knowledge on how $\phi$ and $\psi_{\text {so }}$ depend on the directly tunable magnetic and electric fields, the transmission for polarized current, namely, $T^{0}\left(\varphi_{+}, \omega\right)-T^{0}\left(\varphi_{-}, \omega\right)$, at given electric and magnetic fields can be found. In particular, when the full polarization conditions are met, the total transmission shall satisfy $\left.T\left(\phi, \psi_{\text {so }}, \omega\right)\right|_{\varphi_{\mp}=(2 n-1) \pi}=T^{0}\left(\varphi_{ \pm}, \omega\right)$.

\section{DYNAMICS OF SPIN-DEPENDENT TRANSPORT}

In this section, we discuss the time evolutions of the spin-dependent transport. In the first subsection we study the real time evolution towards a full spinpolarization in the currents, when the two conditions given above are fulfilled. In the second subsection, we turn to the more general situation to investigate the interplay between the $\mathrm{AB}$ and $\mathrm{AC}$ interference effects on the dynamics of spin currents. In the third subsection, we discuss how one can obtain the spin-resolved currents from the total charge currents, using approaches similar to those mentioned in a previous section. 


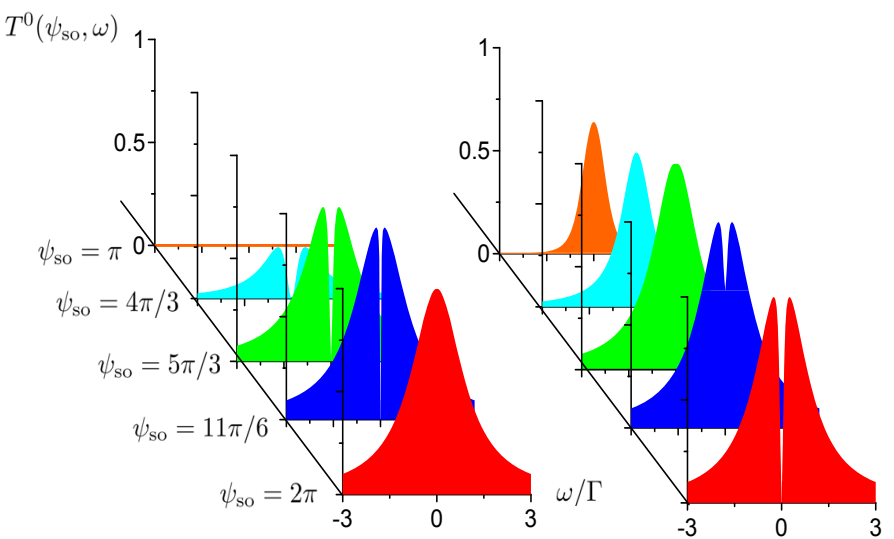

FIG. 3: (color online) The transmission for the effective spinless DQD AB interferometer with the flux set at $\varphi=\psi_{\text {so. The }}$ left panel is for $\delta E=0$ and the right panel is for $\delta E=0.5 \Gamma$. The couplings are $\Gamma_{L}=\Gamma_{R}=\Gamma / 2$ for both panels. These series of stacked plots show $T^{0}\left(\psi_{\mathrm{so}}, \omega\right)$ versus $\omega$ at some specific values of $\psi_{\text {so }}$, as labeled in the figure.

\section{A. Time evolution of full spin polarization in currents}

We have discussed the requirements for generating polarized currents in the steady-state. However, even when those requirements are satisfied, the currents during the transient processes for both of the orthogonal spinors are generally nonvanishing. Using Eq. (22), we explicitly study how the currents for opposite spins along the characteristic directions in each of the electrodes, $I_{\alpha \hat{\mathbf{n}}_{\alpha} ;-}(t)$ and $I_{\alpha \hat{\mathbf{n}}_{\alpha} ;+}(t)$, change in time.

The real-time polarization process is mainly manifested through the evolution of the current $I_{\alpha \hat{\mathbf{n}}_{\alpha} ;-}(t)$, that will eventually decay to zero. The requirement Eq. (29) with Eq. (37) and Eq. (22) leads to

$$
\begin{aligned}
& I_{\alpha \hat{\mathbf{n}}_{\alpha} ;-}(t)=4 \Gamma_{\alpha} \times \\
& \int \frac{d \omega}{2 \pi} f_{\alpha}(\omega) \frac{e^{-\Gamma_{\alpha} t}\left(\Gamma_{\alpha} \cos \omega t+\omega \sin \omega t\right)-\Gamma_{\alpha} e^{-2 \Gamma_{\alpha} t}}{\omega^{2}+\Gamma_{\alpha}^{2}}
\end{aligned}
$$

According to the analysis in Sec. IIC under the condition Eq. (29), the corresponding spinless interferometer, whose current is related to the current carrying the spinor $\left|\hat{\mathbf{n}}_{\alpha} ;-\right\rangle$, has a disconnected effective configuration as that depicted in Fig. 2(a). The result that Eq. (59), as the current for spinor $\left|\hat{\mathbf{n}}_{\alpha} ;-\right\rangle$ on lead $\alpha$, is not affected by anything from the other lead $\bar{\alpha}$, verifies the conclusion in Sec. IC

In contrast to the decay of the currents $I_{\alpha \hat{\mathbf{n}}_{\alpha} ;-}(t)$ toward zero, the time evolution of the currents $I_{\alpha \hat{\mathbf{n}}_{\alpha} ;+}(t)$ generally depends on parameters from both of the leads. At the optimal point $\varphi_{+}=2 m \pi$ leading to $\Gamma_{\varphi_{+}}=\Gamma$, a similar substitution as used for obtaining Eq. (59) results in,

$$
\begin{aligned}
& I_{\alpha \hat{\mathbf{n}}_{\alpha} ;+}(t) \\
= & 4 \Gamma_{\alpha} \int \frac{d \omega}{2 \pi}\left\{f_{\alpha}(\omega) \frac{\Gamma+(\omega \sin (\omega t)-\Gamma \cos (\omega t)) e^{-\Gamma t}}{\omega^{2}+\Gamma^{2}}\right. \\
& \left.-\sum_{\alpha^{\prime}=L, R} \Gamma_{\alpha^{\prime}} f_{\alpha^{\prime}}(\omega) \frac{\left(1+e^{-2 \Gamma t}-2 \cos (\omega t) e^{-\Gamma t}\right)}{\omega^{2}+\Gamma^{2}}\right\} .
\end{aligned}
$$

For this part, the corresponding effective spinless configuration discussed in Sec. [IC is a single-level dot coupled to two reservoirs, as shown in Fig. 2(b). From Eq. (60), one can also see that $I_{\alpha \hat{\mathbf{n}}_{\alpha} ;+}(t \rightarrow \infty) \neq 0$ with nonzero bias, as expected from previous discussions.

Equation (59) indicates that the time for $I_{\alpha \hat{\mathbf{n}}_{\alpha} ;-}(t)$ to reach its steady-state value is mainly determined by the term $e^{-\Gamma_{\alpha} t}$. The smaller $\Gamma_{\alpha}$ is, the slower the full spinpolarization of the current in lead $\alpha$ is reached. On the other hand, one finds from Eq. (60) that the time for $I_{\alpha \hat{\mathbf{n}}_{\alpha} ;+}(t)$ to reach its steady-state value is dominated by the term $e^{-\Gamma t}$. Therefore it is insensitive to the specific values taken by the individual couplings $\Gamma_{L}$ and $\Gamma_{R}$ for a fixed $\Gamma=\Gamma_{L}+\Gamma_{R}$. However, the coupling geometry still affects the magnitude of the full spin-polarized current. In the steady states, Eqs. (48,49) show that the spin-polarized current can be enhanced by having larger value of $\Gamma_{L} \Gamma_{R}$. Note that Eq. (48) is invariant under the exchange of the couplings, $\Gamma_{L} \leftrightarrow \Gamma_{R}$.

In Fig. 4( $(\mathrm{a} 1, \mathrm{a} 2)$ and $(\mathrm{b} 1, \mathrm{~b} 2)$, we demonstrate the effects of the coupling geometry discussed above, specified by different values of $\left(\Gamma_{L}, \Gamma_{R}\right)$, on the time evolution of the spin-resolved currents. The curves in these four plots with the same line styles are with the same pair of couplings $\left(\Gamma_{L}, \Gamma_{R}\right)$, subject to $\Gamma_{L}+\Gamma_{R}=\Gamma$. In Fig. 4 (a1) and (a2), we illustrate the time evolution of $I_{\alpha \hat{\mathbf{n}}_{\alpha} ;-}(t)$. It shows that a smaller $\Gamma_{\alpha}$ leads to a slower decay of the current $I_{\alpha \hat{\mathbf{n}}_{\alpha} ;-}(t)$, thus a slower process of spin-polarization (see the insets for a clearer view). Since we have set $\mu_{L}>\mu_{R}$, more electrons are involved in the left than in the right lead. At later times, this results in generally bigger magnitudes of currents in the left (comparing the magnitudes in the insets of Fig. 4(a1) and (a2).)

In Fig. 4 (b1,b2), the time evolutions of $I_{\alpha \hat{\mathbf{n}}_{\alpha} ;+}(t)$ with $\varphi_{+}=2 m \pi$ are inspected. It shows that different coupling geometries result in similar times for $I_{\alpha \hat{\mathbf{n}}_{\alpha} ;+}(t)$ to reach the corresponding steady-state values. The merging of the curves (blue medium-dashed line merged with green dash-dotted line and black long-dashed line merged with magenta short-dashed line) occur after a time of about $2.5 \Gamma^{-1}$, reaching steady-state values proportional to $\Gamma_{L} \Gamma_{R}$. The maximized spin-polarized current is found with $\Gamma_{L}=\Gamma_{R}=\Gamma / 2$. Comparing Fig. 4 (b) with Fig. 4 (a) insets, one finds that $I_{\alpha \hat{\mathbf{n}}_{\alpha} ;+}(t)$ reaches a stable value generally faster than the full spin-polarization is arrived. This is because the rate for the former, $\Gamma=\Gamma_{L}+\Gamma_{R}$, as a sum of two couplings, is larger than the rate for the 
latter, $\Gamma_{L}$ or $\Gamma_{R}$. The different dependencies of the dynamical processes of the currents $I_{\alpha \hat{\mathbf{n}}_{\alpha} ;-}(t)$ and $I_{\alpha \hat{\mathbf{n}}_{\alpha} ;+}(t)$ on the couplings to the reservoirs, discussed in Sec. IIC, is then illustrated here.

We further investigate the behavior of $I_{\alpha \hat{\mathbf{n}}_{\alpha} ;+}(t)$ when the system is set away from the optimal point $\varphi_{+}=2 m \pi$.

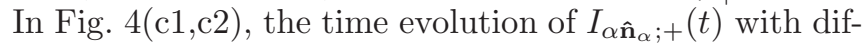
ferent effective fluxes $\varphi_{+}$are plotted. When $\varphi_{+}$is placed away from $2 m \pi$ toward $(2 m+1) \pi$, the steady-state value of this current, proportional to $\cos ^{2}\left(\varphi_{+} / 2\right)$, as inspected from Eq. (48), decreases. Figure $4(\mathrm{c} 1, \mathrm{c} 2)$ also shows that the value of $\varphi_{+}$does not obviously affect the time to approach the steady state but it influences the overall magnitudes of $I_{\alpha \hat{\mathbf{n}}_{\alpha} ;+}(t)$ throughout the time evolution. Note that different values of $\varphi_{+}$are realized by applying different magnetic fluxes and SOI parameters. As long as the choices of the magnetic fluxes and SOI parameters are subjected to Eq. (29c), the time dependence of $I_{\alpha \hat{\mathbf{n}}_{\alpha} ;-}(t)$ remains the same as described by Eq. (59).

\section{B. Dynamics of spin currents}

In the last subsection, we have concentrated on the dynamics under the conditions of reaching fully polarized currents in the steady-state limit. In general when the system deviates from these conditions, both the spinup and the spin-down components of the currents are nonzero and full spin-polarized currents are not attained. In this case, instead of studying separately the currents for spin up and spin down in some specific direction, it is more interesting to simply focus on the spin currents $\mathbf{I}_{\alpha}^{\mathrm{S}}(t)$.

\section{Spin currents due to SOI without the magnetic flux}

We first consider the situation with no applied magnetic flux, namely, $\phi=0$. The effective fluxes are then given by $\varphi_{ \pm}= \pm \psi_{\text {so. }}$. In this case, Eq. (27) becomes (upon the use of Eqs. $(19,21)$ with the aid of $\frac{d}{d t} N_{0}\left(\varphi_{ \pm}, t\right)=I_{L}^{0}\left(\varphi_{ \pm}, t\right)+I_{R}^{0}\left(\varphi_{ \pm}, t\right)$ in Ref. [72] through the identity Eq. (22)),

$$
\begin{aligned}
& \mathbf{I}_{\alpha}^{\mathrm{S}}(t) \cdot \hat{\mathbf{n}}_{\alpha}=-2 \Gamma_{L} \Gamma_{R} \delta E \sin \left(\psi_{\mathrm{so}}\right) \times \\
& \int \frac{d \omega}{2 \pi}\left\{f_{-}(\omega) \frac{1}{\left|\Gamma_{\psi_{\mathrm{so}}}\right|^{2}} \frac{d}{d t}\left[\left|u_{p}(t, \omega)\right|^{2}\right]\right. \\
& \left.\mp f_{+}(\omega)\left[\operatorname{Re}\left(\frac{u_{0}^{*}(t, \omega) u_{p}(t, \omega)}{\Gamma_{\psi_{\mathrm{so}}}}\right)-\Gamma\left|\frac{u_{p}(t, \omega)}{\Gamma_{\psi_{\mathrm{so}}}}\right|^{2}\right]\right\} .
\end{aligned}
$$

where $f_{ \pm}(\omega)=f_{L}(\omega) \pm f_{R}(\omega)$. Here $u_{0, p}(t, \omega)=$ $\int_{0}^{t} d \tau e^{i \omega \tau} u_{0, p}\left(\psi_{\mathrm{so}}, \tau\right)$ where $u_{0, p}\left(\psi_{\mathrm{so}}, \tau\right)$ is equal to $u_{0, p}(\tau)$ of Eq. (16) in Ref. [72] with $\phi$ there replaced by $\psi_{\text {so }}$. The upper sign is for $\alpha=L$ and the lower sign is for $\alpha=R$. Equation (61) shows that if we set $\delta E=0$, then
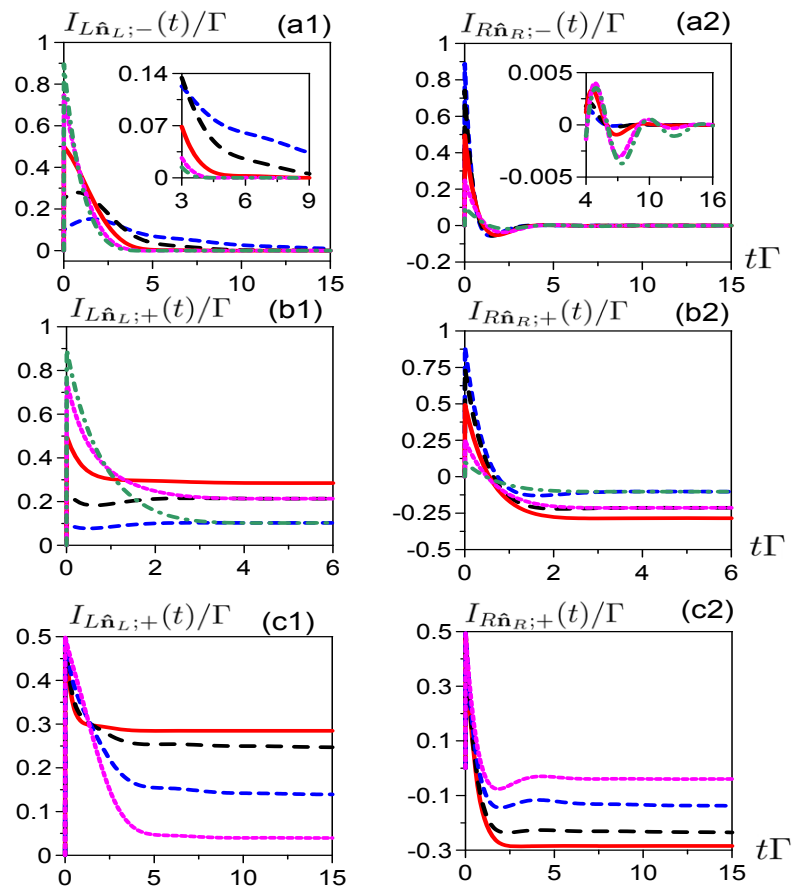

FIG. 4: (color online) Time evolutions of the currents $I_{\alpha \hat{\mathbf{n}}_{\alpha} ;-}(t)$ and $I_{\alpha \hat{\mathbf{n}}_{\alpha} ;+}(t)$ under the conditions of full spin polarizations. In (a1),(a2),(b1),(b2), the blue dashed lines are for $\left(\Gamma_{L}, \Gamma_{R}\right)=(0.1,0.9) \Gamma$, the black long-dashed lines are for $\left(\Gamma_{L}, \Gamma_{R}\right)=(0.25,0.75) \Gamma$, the red solid lines are for $\left(\Gamma_{L}, \Gamma_{R}\right)=(0.5,0.5) \Gamma$, the magenta short-dashed lines are for $\left(\Gamma_{L}, \Gamma_{R}\right)=(0.75,0.25) \Gamma$ and the green dash-dot lines are for $\left(\Gamma_{L}, \Gamma_{R}\right)=(0.9,0.1) \Gamma$. In plots (a1) and $(\mathrm{a} 2), I_{L \hat{\mathbf{n}}_{L} ;-}(t)$ and $I_{R \hat{\mathbf{n}}_{R} ;-}(t)$ are shown for various coupling geometries respectively. The plots (b1) and (b2) respectively are for $I_{L \hat{\mathbf{n}}_{L} ;+}(t)$ and $I_{R \hat{\mathbf{n}}_{R} ;+}(t)$ at the optimal point $\varphi_{+}=2 \pi$. In (c1) and (c2), the time evolutions of the currents $I_{L \hat{\mathbf{n}}_{L} ;+}(t)$ and $I_{R \hat{\mathbf{n}}_{R} ;+}(t)$ are plotted for various $\varphi_{+}$with a fixed coupling geometry $\left(\Gamma_{L}, \Gamma_{R}\right)=(0.5,0.5) \Gamma$. The red solid lines are with $\varphi_{+}=2 \pi$, black long-dashed lines are with $\varphi_{+}=2 \frac{1}{4} \pi$, blue dashed lines are with $\varphi_{+}=2 \frac{1}{2} \pi$ and the magenta short-dashed lines are with $\varphi_{+}=2 \frac{3}{4} \pi$. In all plots, we have set $\delta E=0$, as one of the polarization conditions, and a bias $\mu_{L}=-\mu_{R}=1.25 \Gamma$ is applied at temperature $k_{B} T=\Gamma / 20$. This set of bias and temperature is also assumed in the following figures. The instantaneous rising of the currents to finite values immediately after $t=0$ in these plots is a direct consequence of the wide-band limit. 78

the spin currents remain zero for all time $t$. To generate a non-vanishing spin current, one has to lift up the degeneracy. From Ref. [72], one finds that $\delta E=0$ leads to $I_{\alpha}^{0}\left(\psi_{\mathrm{so}}, t\right)=I_{\alpha}^{0}\left(-\psi_{\mathrm{so}}, t\right)$ for $\alpha=L, R$ and phase rigidity is kept for the underlying spinless system throughout the time. Therefore, generating spin currents by lifting up the degeneracy is equivalent to the temporary breaking of phase rigidity in the spinless DQD interferometer, as pointed out in Ref. [72]. Besides the energy splitting, Eq. (61) also explicitly reveals the necessity of the presence of SOI for the existence of the spin currents, through the term $\sin \left(\psi_{\text {so }}\right)$. When SOI is absent, $\psi_{\text {so }}=0$, then 
$\sin \left(\psi_{\text {so }}\right)=0$, directly leading to $\mathbf{I}_{\alpha}^{\mathrm{S}}(t)=0$. Eq. (61) also shows that the spin currents $\mathbf{I}_{\alpha}^{\mathrm{S}}(t)$ will approach zero at long times. The non-vanishing spin currents can thus only be sustained transiently.

The above discussions show that the magnitudes of the transient spin currents are mainly determined by $\delta E$ and $\sin \left(\psi_{\text {so }}\right)$. In Fig. 5] (a1, a2), we study the effects of various $\delta E$ 's on the time evolutions of the spin currents. The results show that splitting the degeneracy generally enhances the transient spin flow (compare the curves for smaller and bigger $\delta E$ ), as indicated by Eq. (61). Since bigger $\delta E$ also implies faster relaxation, we observe a shorter span of nonzero spin flow with bigger energy splitting. The dependencies of the spin currents on SOI, through the SOI-induced phase $\psi_{\text {so }}$, are presented in Fig. 5] (b1, b2). The transient magnitudes increase with increasing values of $\sin \left(\psi_{\text {so }}\right)$.
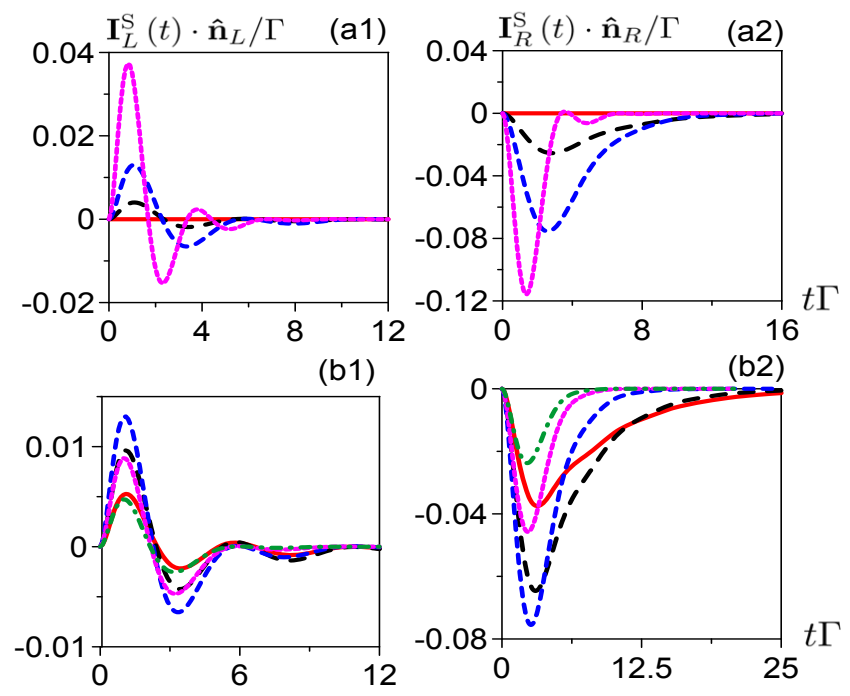

FIG. 5: (color online) Time evolutions of the spin currents. The couplings are set to $\Gamma_{L}=\Gamma_{R}=\Gamma / 2$ here. In plots (a1) and (a2) all curves are with $\psi_{\text {so }}=0.5 \pi$. The red solid lines are for $\delta E=0$, the black long-dashed lines are for $\delta E=0.15 \Gamma$, the blue dashed lines are for $\delta E=0.5 \Gamma$ and the magenta short-dashed lines are for $\delta E=2 \Gamma$. In plots (b1) and (b2) we fix $\delta E=0.5 \Gamma$. The red solid lines are for $\psi_{\text {so }}=0.125 \pi$, the black long-dashed lines are for $\psi_{\text {so }}=0.25 \pi$, the blue dashed lines are for $\psi_{\text {so }}=0.5 \pi$, the magenta short-dashed lines are for $\psi_{\mathrm{so}}=0.75 \pi$ and the green dash-dot lines are for $\psi_{\mathrm{so}}=0.875 \pi$.

\section{Spin currents due to SOI with the magnetic flux}

The above discussions have shown that it is not possible to generate spin currents at degeneracy purely by the act of SOI. Even when the degeneracy between the on-site energies of the DQD is lifted up, the spin currents only survive transiently. The magnetic flux is thus indispensable to sustain non-vanishing spin currents in the long time limit.
The versatility of the combination of the $\mathrm{AB}$ effect and the SOI for attaining various spin currents is demonstrated in Fig. 6 for both $\delta E=0$ and $\delta E \neq 0$. The values of $\phi$ are so chosen that one can attain various distinct results for the spin current. In Fig. 6)(a1,a2), we illustrate that at $\delta E=0$, spin currents can be generated and sustained in the steady states by simultaneously setting $\phi \neq 0$ and $\sin \left(\psi_{\text {so }}\right) \neq 0$. The solution Eq. (27) implies that one can reverse the sign of the spin currents by just adjusting the flux $\phi$, without altering the SOI parameters leading to the changes of $\hat{\mathbf{n}}_{\alpha}$ and $\psi_{\text {so }}$. At degeneracy, the currents $I_{\alpha}^{0}\left(\varphi_{ \pm}, t\right)$ for the effective spinless system depend on the effective flux $\varphi_{ \pm}$only through the term $\cos \varphi_{ \pm}$(see Ref. [72]). Therefore, for all times $t$, the $\operatorname{sign}$ of $\left.\mathbf{I}_{\alpha}^{\mathrm{S}}(t) \cdot \hat{\mathbf{n}}_{\alpha}\right|_{\phi=n \pi+\Delta \phi}$ and that of $\left.\mathbf{I}_{\alpha}^{\mathrm{S}}(t) \cdot \hat{\mathbf{n}}_{\alpha}\right|_{\phi=n \pi-\Delta \phi}$, for $n$ being an arbitrary integer and $\Delta \phi$ being nonzero, are opposite to each other, as indicated in Fig. 6)(a1,a2). By lifting up the degeneracy, the dependence of $I_{\alpha}^{0}\left(\varphi_{ \pm}, t\right)$ on $\varphi_{ \pm}$appears from both of the terms $\cos \varphi_{ \pm}$and $\sin \varphi_{ \pm}$. As a result, the spin currents with $\delta E \neq 0$ are not perfectly antisymmetric with respect to $\phi=n \pi$, as shown by Fig. 6] (b1,b2).

\section{Deduction of the spin-resolved currents from the spin-independent total charge current}

In Sec. IIIB, we have discussed how one can extract the values of $\psi_{\text {so }}$ and $\phi$ at given electric and magnetic fields. With this knowledge in mind, by applying similar procedures, we can also obtain the magnitude of the spin current, $\mathbf{I}_{\alpha}^{\mathrm{S}}(t) \cdot \hat{\mathbf{n}}_{\alpha}$, from the values of the spinindependent total charge current. Explicitly, the spinindependent total charge current, Eq. (24), at given electric and magnetic fields is

$$
I_{\alpha}\left(\phi, \psi_{\mathrm{so}}, t\right)=I_{\alpha}^{0}\left(\varphi_{+}, t\right)+I_{\alpha}^{0}\left(\varphi_{-}, t\right) .
$$

From Eqs. $(19,21)$ in Ref. [2], the current on lead $\alpha$ for the effective spinless DQD system with flux $\varphi$ can be split into two terms,

$$
I_{\alpha}^{0}(\varphi, t)=\bar{I}_{\alpha}^{0}(\varphi, t)+\delta E \sin (\varphi) \bar{I}_{\alpha}^{\gamma}(\varphi, t),
$$

where $\bar{I}_{\alpha}^{0}(\varphi, t)$ and $\bar{I}_{\alpha}^{\gamma}(\varphi, t)$ satisfy,

$$
\bar{I}_{\alpha}^{0}(\varphi, t)=\bar{I}_{\alpha}^{0}(-\varphi, t), \bar{I}_{\alpha}^{\gamma}(\varphi, t)=\bar{I}_{\alpha}^{\gamma}(-\varphi, t) .
$$

Setting zero magnetic field $\phi=0$ in Eq. (62) with the property given by Eq. (63), the dependence of the total charge current on $\psi_{\text {so }}$ becomes

$$
I_{\alpha}\left(\phi=0, \psi_{\mathrm{so}}, t\right)=2 \bar{I}_{\alpha}^{0}\left(\psi_{\mathrm{so}}, t\right)
$$

The dependence of $\bar{I}_{\alpha}^{0}\left(\psi_{\text {so }}, t\right)$ on various $\psi_{\text {so's can thus }}$ be found from the total current $I_{\alpha}\left(\phi=0, \psi_{\text {so }}, t\right)$ under different applied electric fields with zero magnetic flux. The dependence of $\bar{I}_{\alpha}^{\gamma}(\varphi, t)$ on $\varphi$ can be found through the following approach. By fixing the electric field at 
$\psi_{\text {so }}=2 n \pi$, this part of the current is related to the total current and to the part that is already known, $\bar{I}_{\alpha}^{0}(\varphi, t)$, via the relation

$$
2 \delta E \sin (\phi) \bar{I}_{\alpha}^{\gamma}(\phi, t)=I_{\alpha}\left(\phi, \psi_{\text {so }}=2 n \pi, t\right)-2 \bar{I}_{\alpha}^{0}(\phi, t) .
$$

The values of $\bar{I}_{\alpha}^{\gamma}(\phi, t)$ for different $\phi$ 's can thus be deduced from the total current and $\bar{I}_{\alpha}^{0}(\phi, t)$ by applying the corresponding magnetic fields. Knowing $I_{\alpha}^{0}(\varphi, t)$ at various effective fluxes $\varphi$, one can deduce the spin-resolved current by the virtue of Eq. (22).
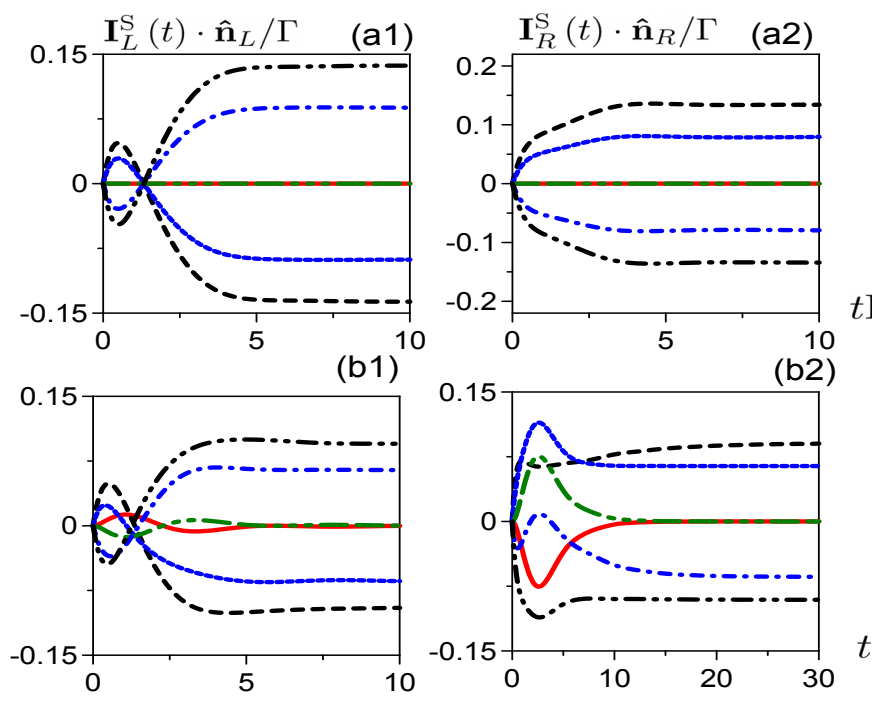

FIG. 6: (color online) Various magnetic flux values are tested for fixed SOI strength. In all plots we let $\psi_{\text {so }}=0.5 \pi$. In plots (a1) and (a2), we set $\delta E=0$. The red solid lines for $\phi=0$ and the dark green dash-dash-dot-dot-dot lines for $\phi=\pi$ are overlapped. The black-dashed lines for $\phi=0.4 \pi$ and the black dash-dot-dot lines for $\phi=1.6 \pi$ differ by a sign. The blue short-dashed lines for $\phi=0.8 \pi$ and the blue dash-dot lines for $\phi=1.2 \pi$ also differ by a sign. In plots (b1) and (b2), the degeneracy is lifted as $\delta E=0.5 \Gamma$ and the same choices of $\phi$ used in (a1) and (a2) are used also here.

\section{SUMMARY AND CONCLUSION}

In this paper, we have explored the real-time dynamics of spin-dependent electron transport through a DQD $\mathrm{AB}$ interferometer with SOI. We have obtained the realtime evolution of the spin-resolved currents, Eq. (28), and the subsequent spin currents Eq. (27). These expressions fully describe the dynamical evolution of the spin-polarizations in the electron transport from initially completely unpolarized interferometers. We have particularly investigated the real-time evolution of the currents towards fully spin-polarized transport. We have also explored the interplay between the SOI and AB interferences in the dynamics of spin flows. Out of these studies, we draw the following conclusions.
(1) The effects of SOI on the transport currents are attributed to the SOI-induced phase $\psi_{\text {so }}$ and the characteristic directions, $\hat{\mathbf{n}}_{L}$ and $\hat{\mathbf{n}}_{R}$. They are fully determined respectively as the eigenvalue and eigenvectors of the unitary spin rotations around the loop. Spin-polarizations of currents in each lead are only developed along their characteristic directions throughout all times. In general, $\hat{\mathbf{n}}_{L} \neq \hat{\mathbf{n}}_{R}$.

(2) The currents carrying electrons with spins along the characteristic directions are equal to the currents of the effective spinless system with the flux replaced by the effective fluxes, as described by Eq. (22). Such connections explicitly reveal that it is the difference between the effective fluxes, $\varphi_{ \pm}=\phi \pm \psi_{\text {so }}$, caused by the SOI, that gives rise to the spin-polarizations.

(3) When fully polarized currents for spin-up electrons occur, the effective spinless system underlying the spindown current corresponds to a disconnected configuration, as shown in Fig. 2(a). Therefore, the decay dynamics of spin-down current in one lead is independent of that in the other lead. In contrast, the dynamics of building spin-up current in one electrode is correlated with that in the other electrode. This essential picture can serve to discern the occurrence of full spin-polarization. One could test it by monitoring the spin-resolved currents in one side while changing parameters of the other side.

(4) The realization of full spin polarization has received considerable attention, as it supplies spin-polarized electron sources and allows the manipulation of flying spin qubits. Therefore it is important to know how to implement fast full spin-polarization and attain the polarized currents of tunable magnitudes. At full polarization condition, we found that increasing the coupling strength to the leads effectively increases the pace toward full spinpolarization. As an interferometer, the magnitude of the resulting currents is largely controlled by the interference phase, which is determined here by the effective fluxes. They are tunable via the magnetic flux and the electric field, affecting the SOI-induced phase.

(5) The connection between the spin-polarized currents and the currents of the effective spinless system provides the underlying physical picture for the working of the spin transport in this DQD AB interferometer. Due to the phase rigidity of the effective spinless system as a twoterminal setup, SOI alone cannot give rise to steady-state spin currents. The indispensable role of the magnetic flux in maintaining nonzero spin currents to the steady-state limit reflects the essence of the interplay between charge and spin interferences. This can be tested by comparing the steady-state spin currents with and without the applied magnetic flux.

(6) Spin-independent total charge currents are readily measurable in experiments. We have shown how one can extract currents of characteristic spins in this system from measurements of the total charge currents at properly chosen electric and magnetic fields. Such measurements can thus be used for testing the properties of the spin-resolved transport concluded above. 
The model we considered in this paper could be constructed from gate-defined QDs made of materials of high carrier density, providing large screening of Coulomb interactions such that electrons are effectively noninteracting. With sufficient orbital level spacing and applying a bias less than such spacing, one can make only a single orbital in each dot participate in the transport. The QDs could be connected to electron reservoirs via gated quantum wires to implement the SOI tunable by the gate electric field. An ongoing experimental issue concerns the possibility to detect spin-polarized electrical currents using only electrical means $\stackrel{79-82}{ }$ Modulating electron transport via interference in ring-like structures with the $A B$ effect $\underline{83,84}$ and spin interference $\frac{12-15}{\underline{15}}$ are of much experimental interest. The analysis of the time evolution of spin-resolved transport for this DQD interferometer could add a momentum to this progressing research direction.

\section{Acknowledgments}

Work at NCKU is partially supported by the National Science Council (NSC) of the ROC, under Contract No. NSC102-2112-M-006-016-MY3, by the Headquarters of University Advancement at the National Cheng Kung University, which is sponsored by the Ministry of Education, Taiwan, ROC and from the National Center for Theoretical Science of NSC and the High Performance Computing Facility in the National Cheng Kung University. Work at Ben Gurion University was supported by grants from the Israel Science Foundation (ISF) and from the U.S.-Israel Binational Science Foundation (BSF).

\section{Appendix A: NEGF formalism for time-dependent transport currents}

Here we summarize formulations for studying nonequilibrium electron transport through a class of nanoelectronic structures. In order to consider spin-dependent dynamics, here we label both the charge and the spin degrees of freedom explicitly. We also assume that electron reservoirs are free from SOI. The Hamiltonian of the total system is then given by Eq. (10), where the Hamiltonian of the central area is generally

$$
\mathcal{H}_{\mathrm{S}}=\sum_{i j, \sigma \sigma^{\prime}} E_{i \sigma, j \sigma^{\prime}} a_{i \sigma}^{\dagger} a_{j \sigma^{\prime}}
$$

with $i, j$ labeling orbital states and $\sigma, \sigma^{\prime}$ denoting the spins. The Hamiltonian for sum of electron reservoirs is Eq. (3), with $\alpha$ running over all considered electrodes. The tunneling between the central system and the leads is described by Eq. (4). In all the above equations, the spins are quantized along an arbitrary direction.

The spin-resolved transient current at time $t$ is defined by,

$$
I_{\alpha \sigma}(t)=-\frac{d}{d t} \operatorname{tr}_{\text {tot }}\left[\mathcal{N}_{\alpha, \sigma} \rho_{\text {tot }}(t)\right]
$$

where

$$
\mathcal{N}_{\alpha, \sigma}=\sum_{\boldsymbol{k} \in \alpha} c_{\alpha \boldsymbol{k} \sigma}^{\dagger} c_{\alpha \boldsymbol{k} \sigma},
$$

is the total particle number operator for spin $\sigma$ in lead $\alpha$ and $\rho_{\text {tot }}(t)$ is the total density matrix of the central system plus the electron reservoirs at time $t$. Here $\operatorname{tr}_{\text {tot }}$ denotes the trace over the total system.

As usual, we assume ${ }^{\frac{76}{6}}$ that at the initial time $t=$ $t_{0}$, the central system is decoupled from the leads, and the leads are at thermal equilibrium with the chemical potential $\mu_{\alpha \sigma}$ and the temperature $T_{\alpha \sigma}$ for electron with spin $\sigma$ in lead $\alpha$, whose Fermi distribution function is given by,

$$
f_{\alpha \sigma}(\omega)=1 /\left[e^{\left(\omega-\mu_{\alpha \sigma}\right) / k_{B} T_{\alpha \sigma}}+1\right],
$$

where $k_{B}$ is the Boltzmann constant. If the central area initially contains no excess electrons, then the real-time current carrying electrons of spin $\sigma$ from lead $\alpha$ in terms of Keldysh NEGF reads 57.77

$$
\begin{aligned}
& I_{\alpha \sigma}(t)=-2 \operatorname{Re} \operatorname{Tr} \\
& \int_{t_{0}}^{t} d \tau\left\{\boldsymbol{\Sigma}_{\alpha \sigma}^{r}(t, \tau) \mathbf{G}^{<}(\tau, t)+\boldsymbol{\Sigma}_{\alpha \sigma}^{<}(t, \tau) \mathbf{G}^{a}(\tau, t)\right\} .
\end{aligned}
$$

Throughout the paper, we use units in which $\hbar=e=1$. One can also derive the same current formula through a density matrix formalism, $\frac{67}{\underline{T}}$ as used in Sec. IID and the two expressions can be identified via Eqs.(35/36). The retarded and the lesser self-energies are

$$
\begin{aligned}
& \boldsymbol{\Sigma}_{\alpha \sigma}^{r}(t, \tau)=-i \theta(t-\tau) \int \frac{d \omega}{2 \pi} \boldsymbol{\Gamma}^{\alpha \sigma}(\omega) e^{-i \omega(t-\tau)}, \\
& \boldsymbol{\Sigma}_{\alpha \sigma}^{<}(t, \tau)=i \int \frac{d \omega}{2 \pi} f_{\alpha \sigma}(\omega) \boldsymbol{\Gamma}^{\alpha \sigma}(\omega) e^{-i \omega(t-\tau)}
\end{aligned}
$$

respectively, with $\theta$ being the step function. They are defined via the level-broadening function,

$$
\left[\boldsymbol{\Gamma}^{\alpha \sigma}(\omega)\right]_{i \sigma^{\prime}, j \sigma^{\prime \prime}}=2 \pi \sum_{\boldsymbol{k} \in \alpha} V_{i \sigma^{\prime}, \alpha \boldsymbol{k} \sigma} V_{j \sigma^{\prime \prime}, \alpha \boldsymbol{k} \sigma}^{*} \delta\left(\omega-\epsilon_{\alpha \boldsymbol{k}}\right),
$$

The retarded and the advanced Green functions are defined by

$$
\begin{aligned}
& {\left[\mathbf{G}^{r}(t, \tau)\right]_{i \sigma, j \sigma^{\prime}}=-i \theta(t-\tau)\left\langle\left\{a_{i \sigma}(\tau), a_{j \sigma^{\prime}}^{\dagger}(t)\right\}\right\rangle,} \\
& {\left[\mathbf{G}^{a}(\tau, t)\right]_{i \sigma, j \sigma^{\prime}}=i \theta(t-\tau)\left\langle\left\{a_{i \sigma}(\tau), a_{j \sigma^{\prime}}^{\dagger}(t)\right\}\right\rangle .}
\end{aligned}
$$

They are related by $\mathbf{G}^{a}(\tau, t)=\left[\mathbf{G}^{r}(t, \tau)\right]^{\dagger}$. The retarded Green function follows the equation

$$
\left[i \partial_{t}-\boldsymbol{E}\right] \mathbf{G}^{r}(t, \tau)-\int_{\tau}^{t} d \tau^{\prime} \boldsymbol{\Sigma}^{r}\left(t, \tau^{\prime}\right) \mathbf{G}^{r}\left(\tau^{\prime}, \tau\right)=\delta(t-\tau),
$$


and the lesser Green function is given by

$\mathbf{G}^{<}(\tau, t)=\int_{t_{0}}^{\infty} d \tau^{\prime} \int_{t_{0}}^{\infty} d \tau^{\prime \prime} \mathbf{G}^{r}\left(\tau, \tau^{\prime}\right) \boldsymbol{\Sigma}^{<}\left(\tau^{\prime}, \tau^{\prime \prime}\right) \mathbf{G}^{a}\left(\tau^{\prime \prime}, t\right)$

Here $\boldsymbol{E}$ is the energy matrix of the central system while

$$
\begin{aligned}
& \boldsymbol{\Sigma}^{r}\left(\tau, \tau^{\prime}\right)=\sum_{\alpha \sigma} \boldsymbol{\Sigma}_{\alpha \sigma}^{r}\left(\tau, \tau^{\prime}\right), \\
& \boldsymbol{\Sigma}^{<}\left(\tau, \tau^{\prime}\right)=\sum_{\alpha \sigma} \boldsymbol{\Sigma}_{\alpha \sigma}^{<}\left(\tau, \tau^{\prime}\right)
\end{aligned}
$$

are sums of individual self-energies induced by coupling to each of the leads. In the definitions Eqs. (A6A7], the time-dependent field operators are those in the Heisenberg picture and the bracket denotes the average over the initial state, $\langle\cdot\rangle=\operatorname{tr}_{\text {tot }}\left(\cdot \rho_{\text {tot }}\left(t_{0}\right)\right)$.

By specifying the level-broadening function, Eq. (A5c), and therefore the self-energies, Eq. (A5), one can substitute them into Eqs. A6, A7) for solving the Green functions in the time domain. The real time currents can then be found by substituting these Green functions and self-energies into Eq. (A4).
* Electronic address: aaharonyaa@gmail.com

† Electronic address: wzhang@mail.ncku.edu.tw

1 Y. Aharonov and D. Bohm, Phys. Rev. 115, 485 (1959).

2 Y. Aharonov and A. Casher, Phys. Rev. Lett. 53, 319 (1984).

3 M. Büttiker, Y. Imry, and R. Landauer, Phys. Lett. A 96, 365 (1983).

${ }^{4}$ M. Buttiker, Y. Imry, and M. Ya. Azbel, Phys. Rev. A 30, 1982 (1984)

5 Y. Gefen, Y. Imry, and M. Ya. Azbel, Phys. Rev. Lett. 52, 129 (1984).

6 A. G. Aronov, Yu. V. Sharvin, Rev. Mod. Phys. 59, 755 (1987).

7 A. Yacoby, M. Heiblum, V. Umansky, H. Shtrikman, and D. Mahalu, Phys. Rev. Lett. 73, 3149 (1994).

8 A. Yacoby, M. Heiblum, D. Mahalu, and H. Shtrikman, Phys. Rev. Lett. 74, 4047 (1995).

9 R. Schuster, E. Buks, M. Heiblum, D. Mahalu, V. Umansky, and H. Shtrikman, Nature (London) 385, 417 (1997).

10 E. Buks, R. Schuster, M. Heiblum, D. Mahalu, and H. Shtrikman, Nature (London) 391, 871 (1998).

11 H. Mathur and A. D. Stone, Phys. Rev. Lett. 68, 2964 (1992).

12 M. König, A. Tschetschetkin, E. M. Hankiewicz, J. Sinova, V. Hock, V. Daumer, M. Schäfer, C. R. Becker, H. Buhmann, and L. W. Molenkamp, Phys. Rev. Lett. 96, 076804 (2006).

13 T. Bergsten, T. Kobayashi, Y. Sekine, and J. Nitta, Phys. Rev. Lett. 97, 196803 (2006).

14 F. Nagasawa, J. Takagi, Y. Kunihashi, M. Kohda, and J. Nitta, Phys. Rev. Lett. 108, 086801 (2012).

15 F. Nagasawa, D. Frustaglia, H. Saarikoski, K. Richter, J. Nitta, Nat. Commun. 4, 2526 (2013).

16 I. Žutić, J. Fabian, and S. Das Sarma, Rev. Mod. Phys. 76, 323 (2004).

17 S. Datta and B. Das, Appl. Phys. Lett. 56, 665 (1990).

18 A. Oiwa, Y. Mitsumori, R. Moriya, T. Słupinski, and H. Munekata, Phys. Rev. Lett. 88, 137202 (2002).

19 B. T. Jonker, G. Kioseoglou, A. T. Hanbicki, C. H. Li, and P. E. Thompson, Nat. Phys. 3, 542 (2007).

${ }^{20}$ P. LeClair, J. K. Ha, H. J. M. Swagten, J. T. Kohlhepp, C. H. van de Vin, and W. J. M. de Jonge, Appl. Phys. Lett. 80, 625 (2002).

21 T. S. Santos and J. S.Moodera, Phys. Rev. B 69, 241203(R) (2004).

22 M. Gajek, M. Bibes, A. Barthélémy, K. Bouzehouane, S.
Fusil, M. Varela, J. Fontcuberta, and A. Fert, Phys. Rev. B 72, 020406(R) (2005).

23 U. Lüders, M. Bibes, K. Bouzehouane, E. Jacquet, J.P. Contour, S. Fusil, J.-F. Bobo, J. Fontcuberta, A. Barthélémy, and A. Fert, Appl. Phys. Lett. 88, 082505 (2006).

24 G. Schmidt, D. Ferrand, L. W. Molenkamp, A. T. Filip, and B. J. van Wees, Phys. Rev. B 62, R4790 (2000).

${ }^{25}$ K. Ando, S. Takahashi, J. Ieda, H. Kurebayashi, T. Trypiniotis, C. H. W. Barnes, S. Maekawa, and E. Saitoh, Nature Mater. 10, 655 (2011).

26 G. Dresselhaus, Phys. Rev. 100, 580 (1955).

27 E. I. Rashba, Fiz. Tverd. Tela (Leningrad) 2, 1224 (1960) [Sov. Phys. Solid State 2, 1109 (1960)]; Y. A. Bychkov and E. I. Rashba, J. Phys. C 17, 6039 (1984).

28 J. Nitta, T. Akazaki, H. Takayanagi, and T. Enoki, Phys. Rev. Lett. 78, 1335 (1997); T. Koga, J. Nitta, T. Akazaki, and H. Takayanagi, ibid. 89, 046801 (2002).

29 T. Koga, Y. Sekine, and J. Nitta, Phys. Rev. B 74, 041302 (2006).

30 J. Nitta, F. E. Meijer, and H. Takayanji, Appl. Phys. Lett. 75, 695 (1999).

31 D. Frustaglia and K. Richter, Phys. Rev. B 69, 235310 (2004).

32 B. Molnár, F. M. Peeters, and P. Vasilopoulos, Phys. Rev. B 69, 155335 (2004).

33 R. Citro, F. Romeo, and M. Marinaro, Phys. Rev. B 74, 115329 (2006).

${ }^{34}$ U. Aeberhard, K. Wakabayashi, and M. Sigrist, Phys. Rev. B 72, 075328 (2005).

35 V. Moldoveanu and B. Tanatar, Phys. Rev. B 81, 035326 (2010).

36 D. Bercioux, D. Frustaglia, and M. Governale, Phys. Rev. B 72, 113310 (2005).

37 V. M. Ramaglia, V. Cataudella, G. De Filippis, and C. A. Perroni, Phys. Rev. B 73, 155328 (2006).

38 M. J. van Veenhuizen, T. Koga, and J. Nitta, Phys. Rev. B 73, 235315 (2006).

39 P. Földi, O. Kálmán, M. G. Benedict, and F. M. Peeters, Phys. Rev. B 73, 155325 (2006).

40 F. Chi and J. Zheng, Appl. Phys. Lett. 92, 062106 (2008).

41 N. Hatano, R. Shirasaki, and H. Nakamura, Phys. Rev.A 75, 032107 (2007).

42 S.-H. Chen and C.-R. Chang, Phys. Rev. B 77, 045324 (2008)

43 A. Aharony, Y. Tokura, G. Z. Cohen, O. Entin-Wohlman, 
and S. Katsumoto, Phys. Rev. B 84, 035323 (2011).

44 F. Chi, J.-L. Liu, and L.-L. Sun, J. Appl. Phys. 101, 093704 (2007).

${ }^{45}$ F. Chi, X. Yuan, and J. Zheng, Nanoscale Res. Lett. 3, 343 (2008).

${ }^{46}$ H.-T. Yin, X.-J. Liu, L.-F. Feng, T.-Q. Lu, and H. Li, Phys. Lett. A 374, 2865 (2010).

47 K.-W. Chen, Y.-H. Su, S.-H. Chen, C.-L. Chen, and C.-R. Chang, Phys. Rev. B 88, 035443 (2013).

48 P. M. Shmakov, A. P. Dmitriev, and V. Yu. Kachorovskii, Phys. Rev. B 85, 075422 (2012); P. M. Shmakov, A. P. Dmitriev, and V. Yu. Kachorovskii, ibid, 87, 235417 (2013).

49 M. Pletyukhov, V. Gritsev, and N. Pauget, Phys. Rev. B 74, 045301 (2006).

50 A. M. Lobos and A. A. Aligia, Phys. Rev. Lett. 100, 016803 (2008)

51 R. Citro and F. Romeo, Phys. Rev. B 73, 233304 (2006).

${ }^{52}$ P. Földi, M. G. Benedict, O. Kálmán, and F. M. Peeters, Phys. Rev. B 80, 165303 (2009).

53 J. Bylander, T. Duty, and P. Delsing, Nature (London) 434, 361 (2005).

54 T. Fujisawa, T. Hayashi, and S. Sasaki, Rep. Prog. Phys. 69, 759 (2006).

${ }^{55}$ G. Fève, A. Mahé, J.-M. Berroir, T. Kontos1, B. Plaçais, D. C. Glattli, A. Cavanna, B. Etienne, Y. Jin, Science 316, 1169 (2007).

${ }_{57}$ M. Cini, Phys. Rev. B 22, 5887 (1980).

57 A.-P. Jauho, N. S. Wingreen, and Y. Meir, Phys. Rev. B 50, 5528 (1994).

58 G. Stefanucci and C.-O. Almbladh, Phys. Rev. B 69, 195318 (2004).

59 F. B. Anders and A. Schiller, Phys. Rev. Lett. 95, 196801 (2005).

60 S. Kurth, G. Stefanucci, C.-O. Almbladh, A. Rubio, and E. K. U. Gross, Phys. Rev. B 72, 035308 (2005).

61 J. Maciejko, J. Wang, and H. Guo, Phys. Rev. B 74, 085324 (2006).

${ }^{62}$ X. Zheng, F. Wang, C. Y. Yam, Y. Mo, and G. H. Chen, Phys. Rev. B 75, 195127 (2007).

${ }^{63}$ L. Mühlbacher and E. Rabani, Phys. Rev. Lett. 100, 176403 (2008).

64 T. L. Schmidt, P. Werner, L. Mühlbacher, and A. Komnik, Phys. Rev. B 78, 235110 (2008).

65 J. S. Jin, X. Zheng, and Y. J. Yan, J. Chem. Phys. 128, 234703 (2008).

66 M. W. -Y. Tu and W. -M. Zhang, Phys. Rev. B 78, 235311 (2008); M. W. -Y. Tu, M.-T. Lee, and W. -M. Zhang, Quantum Inf. Processing (Springer) 8, 631 (2009).

67 J. S. Jin, M. W. -Y. Tu, W. -M. Zhang, and Y. J. Yan, New J. Phys. 12, 083013 (2010).

68 D. Segal, A. J. Millis, and D. R. Reichman, Phys. Rev. B 82, 205323 (2010).
69 H. Cruz and D. Luis, J. Phys.: Conf. Ser. 99, 012004 (2008).

70 E. Perfetto, G. Stefanucci, and M. Cini, Phys. Rev. B 78, 155301 (2008).

71 M. W.-Y. Tu, W.-M. Zhang, and J. S. Jin, Phys. Rev. B 83, 115318 (2011); M. W.-Y. Tu, W.-M. Zhang and F. Nori, ibid, 86195403 (2012).

72 M. W.-Y. Tu, W.-M. Zhang, J. S. Jin, O. Entin-Wohlman, and A. Aharony, Phys. Rev. B 86, 115453 (2012).

73 Y. Oreg and O. Entin-Wohlman, Phys. Rev. B 46, 2393 (1992).

74 Y. Meir, Y. Gefen, and O. Entin-Wohlman, Phys. Rev. Lett. 63, 798 (1989).

75 In Ref. 43], the authors diagonalized the quantities, $\mathbf{W} \mathbf{W}^{\dagger}$ and $\mathbf{W}^{\dagger} \mathbf{W}$, where $\mathbf{W}=\gamma_{b} U_{b}+\gamma_{c} U_{c}$ (using the notations in that paper), in which $\gamma_{b, c}$ are some real scalar coefficients relating to couplings to the leads. The unitary spin rotations are identified to be $U_{b}=U^{L 1} U^{1 R}$ and $U_{c}=U^{L 2} U^{2 R}$. The spin rotations around the loop commute with these quantities, namely, $\left[\mathbf{W} \mathbf{W}^{\dagger}, U^{L}\right]=0$ and $\left[\mathbf{W}^{\dagger} \mathbf{W}, U^{R}\right]=0$. Therefore the eigenstates of $\mathbf{W} \mathbf{W}^{\dagger}\left(\mathbf{W}^{\dagger} \mathbf{W}\right)$ obtained there are also those of $U^{L}\left(U^{R}\right)$ as needed here.

76 A. J. Leggett, S. Chakravarty, A. T. Dorsey, M. P. Fisher, A. Garg, and W. Zwerger, Rev. Mod. Phys. 59, 1 (1987).

77 H. Haug and A.-P. Jauho, in Quantum Kinetics in Transport and Optics of Semiconductors, Springer Series in Solid-State Sciences, 2nd ed. (Springer-Verlag, Berlin, 2008), Vol. 123.

78 The wide-band limit means that there is an infinite number of levels in the reservoir that couple equally strong to the discrete levels in the quantum dot. So every level in the reservoir within this band of infinite width tries to tunnel to the discrete levels, causing an immediate finite current when the contact begins. This feature of wide-band levelbroadening function is also documented 57,64 We demonstrate all numerical results in the wide-band limit here for simplicity and for staying connected with our previous work ${ }^{72}$

79 T. Otsuka, E. Abe, Y. Iye, and S. Katsumoto, Phys. Rev. B 79, 195313 (2009).

80 P. Debray, S. M. S. Rahman, J. Wan, R. S. Newrock, M. Cahay, A. T. Ngo, S. E. Ulloa, S. T. Herbert, M. Muhammad, and M. Johnson, Nature Nanotech. 4, 759 (2009).

81 S. Kim, Y. Hashimoto, Y. Iye, and S. Katsumoto, J. Phys. Soc. Jpn. 81, 054706 (2012).

82 T.-M. Chen, M. Pepper, I. Farrer, G. A. C. Jones, and D. A. Ritchie, Phys. Rev. Lett. 109, 177202 (2012).

83 T. Hatano, T. Kubo, Y. Tokura, S. Amaha, S. Teraoka, and S. Tarucha, Phys. Rev. Lett. 106, 076801 (2011).

84 J. Verduijn, R. R. Agundez, M. Blaauboer and S. Rogge, New J. Phys. 15, 033020 (2013). 